
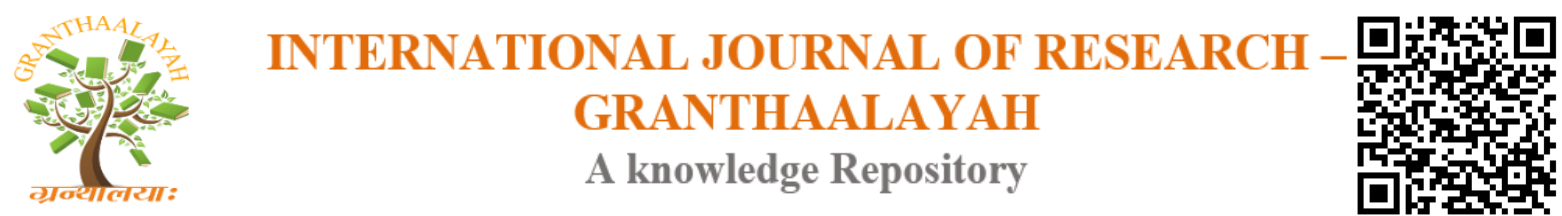

Science

\title{
BEE FLORAL CALENDAR OF CULTIVATED AND WILD PLANTS AVAILABLE IN DIFFERENT AGROECOSYTEMS OF CHITWAN, NEPAL
}

\author{
Shiva P. Rijal' ${ }^{1}$, Resam B. Thapa ${ }^{2}$, Moha D. Sharma ${ }^{3}$, Shrawan K. Sah ${ }^{4}$, Yubak Dhoj GC \\ ${ }^{1} \mathrm{Ph} . \mathrm{D}$. scholar of Institute of Agriculture and Animal Science (IAAS), Tribhuvan University \\ (TU), Nepal \\ ${ }^{2}$ Professor, IAAS, TU, Kathmandu, Nepal \\ ${ }^{3,4}$ Professor, AFU, Rampur, Nepal \\ ${ }^{5}$ Secretary, Ministry of Agriculture and Livestock Development, Singh Durbar, Kathmandu, \\ Nepal
}

\begin{abstract}
Beekeeping is one of the promising enterprises for economically poor farmers in Nepal but beekeeping farmers lack flora calendar for pollination, bee foraging, and honey production. Therefore, a study was conducted visiting farmers' fields every 15-20 days during 2012-2013 to monitor the common plant species visited by bees, which were considered as bee forage plants categorized as major, and minor sources of pollen and/or nectar. Relevant information was also gathered through key informant interviews and group discussions. During the study period a total of 252 plant species were recorded and their floral calendar prepared. In the study area. The main species identified were: rice, Oryza sativa L.; maize, Zea mays L.; buckwheat, Fagopyrum esculentum Moench.; rapeseed, Brassica campestris L.; sesame, Sesamum orientale L.; litchi, Litchi chinensis Sonner; and cucurbits (bottle gourd, Lagenaria siceraria (Molina) Standl; sponge gourd, Luffa cylindrica (L.) Roem.; bitter gourd, Momordica charantia L.), Leucas (gumpate), Leucas lanata Benth.; Butternut (chiuri), Bassia butyracea Roxb.; Pogostemon (rudilo), Pogostemon glaber Benth.; guava, Pisum sativum L; Sisoo, Dalbergia sissoo Roxb.; Throughwort (Banmara), Eupatorium sp.; silk tree (Padke), Albizia julibrissin Durazz ; Terminalia (Saj) Terminalia bellirica (Geartn.) Roxb. and Murraya (kadipatta) Murraya koenigii (L.) Spreng. Species of Brassica, Pogestemon, Bassia, Citrus, and Artemisia, Pisum, Ipomoea and Eupatorium species were some of the important plants which bloomed during winter. And, Lagerstroemia sp., Impatiens sp, Sesamum indicum L., Zea mays L., Albizia sp. and many cucurbits bloomed during rainy season. Fagopyrum esculentum Moench, Brassica campestral L, Lagerstroemia indica L, Bombax ceiba L, Albizia julibrissin Durazz., Eugenia formosa Wall., Shorea robusta, Guartn. Upatorium grandulosum, Pogostemon glaber Benth. Terminalia alata Heyne ex.Roth, Murraya koenigii (L.) Spreng Sesamum orientale L. Dalbergia sissoo Roxb. were the major bee flora both rich in nectar and pollen.
\end{abstract}

Keywords: Bee forage; honeybees; buckwheat; rapeseed; Sisoo; Pogostemon. 
Cite This Article: Shiva P. Rijal, Resam B. Thapa, Moha D. Sharma, Shrawan K. Sah, and Yubak Dhoj GC. (2018). "BEE FLORAL CALENDAR OF CULTIVATED AND WILD PLANTS AVAILABLE IN DIFFERENT AGROECOSYTEMS OF CHITWAN, NEPAL." International Journal of Research - Granthaalayah, 6(11), 222-245.

\section{Introduction}

Nepal is rich in plant and insect diversities. The wide diversities of crops and other plants benefit from pollination services of insects particularly bees, which result in one-third of the total human diet with pollination value worth of 143 times higher than honey production in the world (Mishra, 1997/98). The value of insect pollination for worldwide agricultural production is estimated at 153 billion, which represents $9.5 \%$ of the value of the world agricultural production used for human food in 2005 (Gallai et al., 2009). The average global value of pollination system is $\$ 117 \mathrm{ha}^{-1} \mathrm{yr}^{-}$ ${ }^{1}$ (Costanza et al., 1997). Crop pollination yield with enhanced quality in entomophilous orchard fruit crops (Garratt et al., 2013), in field crops (Bommarco, 2012), and small fruits and vegetables (Andersson, 2012; Roselino et al., 2009; Hogendoorn et al., 2014; Roldan, 2006; Isaacs, 2010). Some crops entirely depend on insect pollination for seed and fruit production, whereas others benefit with higher yields, better quality produce and uniform maturation (Breeze et al., 2011).

Chitwan district lies in the southern part of Narayani zone in central Nepal. This district enjoys a sub-tropical climate with a unique ecosystem of significant value to the world (i.e. Chitwan National Park). Approximately, $70 \%$ of the National Park vegetation is sal (Shorea robusta) forest, grassland (20\%), riverine forest (7\%), and sal with Chirpine (3\%). The riverine forests consist mainly of khair (Acacia catechu (L.f.) Wilid. sissoo (Dalbergia sissoo Roxb.) and simal (Bombax ceiba Lin.). The grassland forms a diverse and complex community with over 50 species (Dangol, 2000). This district is well acquainted as granary of Nepal. However, high pressure of migrant from different district of Nepal in Chitwan resulted in negative impact, i.e. change in biodiversity and vegetation composition (Dangol, 2000). Declining of pollinators has been noticed by different researchers (Pokhrel 2005; Biesmeijer et al., 2006; Cameron et al., 2011; Goulson et al., 2008; Englesdorp et al., 2008). Wilson (1988) estimated that 0.2-0.3\% of all species are lost every year in globe. Range of 5-10\% of the tropical forest species may become extinct within the next 30 years (UNEP, 1993). In the district, there are no in-depth records of bee flora in pollination and beekeeping perspectives, which have long lasting effect on the livelihood of the people living in the area.

To maintain diversity of flora, self-incompatible and cross-pollinated crops require efficient pollination services of honeybees and other pollinators, and also self-pollinated crops benefit from insect by showing their hybrid vigor without any desertion in their innate properties of fruits and seeds (Thapa, 2006). The flowering plants of an area having good value as bee pasture are necessary to maintain bee colonies (Baptist and Punchihewa, 1980). Hence, every beekeeper must be familiar with the bee floral resources near/ or around his/her apiary for successful beekeeping and also bee fauna greatly influence crop pollination and reward hive production.

All pollinator species do not respond equally to land use change (Williams et al., 2010; Winfree et al., 2011). The flowering crop field has been known as an important driver for pollinator community composition, and the landscape context needs to be considered when linking land use 
to pollination provisioning and benefits to field crops (Bartomeus et al., 2014). Delaplane et al. (2010) revealed that in planning a bee pasture, it is important to choose a collection of plants that will produce unbroken succession of bloom throughout the season. Furthermore, seasonal changes in the vegetation patterns, the foraging behavior of bees, and the manner in which the honeybee colonies interact with their floral environment are the major events which determine the best time to carry out bee husbandry practice or prepare for the honey seasons and food shortage periods with feeding management as well. Different studies (Partap and Partap, 2002; Thapa, 2002, Adhikari and Ranabhat, 2003) reveal that level of knowledge about biodiversity conservation, pollination and pollinators in Nepalese farmers are inadequate. Hence, this study was carried out to identify the common bee flora in the study areas, estimate their seasonal abundance and diversity, prepare flowering calendar and identify the nectar and/or pollen producing sources for potential beekeeping by the farmers.

\section{Materials and Methods}

\section{Study Area}

Four study areas with different ecosystems in Chitwan, i.e. Megauli (semi-natural), Fulbari (organic), Jutpani (intensive agriculture) and Siddhi (Hill, semi-natural) Village Development Committees (VDCs) were selected for this study. Meghauli VDC have $25 \mathrm{~km}$ long community forest is another Eco-Park and medicinal herbal farming in the buffer zone as attractions of the study area, which is an example of good balance between human settlements and natural environments. Fulbari VDC occupies 1388 ha of land and family households here, chiefly are engaged in agricultural activities, mainly organic agriculture. Jutpani, the other VDC, has 1200 ha land with 767ha cultivable land (Bariland 621 ha, khetland 146 ha.) and 50 ha of forest and others. This site is dominated by intensive agriculture with the use of hybrid seeds, chemical fertilizer and pesticides. Beekeeping is a flourishing business there, however spraying of pesticide in rapeseed and vegetables are threats to beekeeping and environment. Sidhhi VDC occupies 3403ha of land in which the cultivable land is 3291.2 hectares and most of land is undulated and sloppy. This VDC is rich in flora and fauna, since the area other than cultivated is under leasehold forest and community forests' control.

Direct observation of flowering plants was done every 20 days during 2012/013. Each study visit served as pseudo replicates for the site and all observations were made between 7:00-18:00 hours in winter and autumn; and 7:00-18:30 hours in summer season. Honey bee foraging plant were determined with visit of honeybee workers on its flowers for 10 minutes (Silveira, 2004). The observation on nectar and pollen source was based on activities performed by honeybees on different flowers, i.e. honeybees with their activity of extending their proboscis into the flowers were considered as nectar source and bees carrying pollen on their hind legs were determined as pollen source plants ((Mbah and Amao, 2004; Bista and Shivakoti, 2000-2001). Most plants visited by honeybees were identified in the field by comparing with the published reports (Partap, 1997; Polunin and Stainton, 1997; Shrestha, 1998; Thapa, 2006) and those unknown plants were identified with the help of the experts from Tribhuvan University (TU), Agriculture and Forestry University (AFU) and Nepal Agriculture Research Council (NARC). Plants were categorized either rich in pollen $(\mathrm{P} 1, \mathrm{P} 2)$ or in nectar $(\mathrm{N} 1, \mathrm{~N} 2)$ or combination of two in different levels (Partap, 1997). For scaling up beekeeping, the area of agricultural bee forage (in hectare) and potential foraging bee colonies with minimum of two hives per hectare were estimated as potentiality of bee 
forage supporting honeybee colonies in those areas (Partap and Partap, 1997). Honey potential is the estimated weight $(\mathrm{kg})$ of honey that can be obtained in the course of a season from one hectare of land covered with the plant assuming optimal condition (Crane, 1975), which varies from species to species and also according to climatic and soil condition (Partap, 1997).

Key informant interview and focus group discussion were also organized once in each study area purposively selecting the communities, such as pollination farmer groups, local leaders, buffer zone technicians, bee technician, and other individuals, who are believed to be knowledgeable about bee flora of the study sites. The secondary data included the existing research literature. Related websites of GOs and INGOs, such as, ICIMOD, IAAS, AFU, CBS, CABI, IUCN, and ISSG etc. were consulted to review literature on biological characteristics of the plants. Data were managed in Microsoft Excel and analyzed using descriptive statistics for frequency, density, flowering duration etc.

\section{Results and Discussion}

\section{Crops and Wild Plants Distributed in The Study Areas}

The dominant crops benefited from cross pollination grown in Megauli (semi-natural site) were Chilli, Buckwheat, Broad leaf mustard, Bottle gourd, Mango, Papaya and Rice were crops benefitted by pollination, Coriander, Bitter gourd, Chilli, Sunflower, Litchi, Pumpkin, Sponge gourd, Mustard and Rice in Fulbari (organic site) Bitter gourd, Marigold, Wheat, Okra, Banana, Cowpea, Maize and Rice were the pollination dependent crops in Jutpani (Intensive agriculture site) and Citrus, Maize, Rice and Vegetables in Siddh ( hill site). In wild plants Leucas, Murraya (kadipatta), Pogostemon (rudilo) Sisoo, Throughwort (Banmara) available in Megauli ; silk tree (Padke), Pogestemon, Artemisia, Eupatorium, and Termenelia in Jutpani Butternut (chiuri), wild Sesamum Albizia sp. in Siddhi, Lagerstroemia indica L, Bombax Albizia,Sissoo, Upatorium in Fulbari. The distribution of crops and wild plants in groups is shown in Table 1.

Table 1: Number of bee forage species available in different land use sites in 2012/013

\begin{tabular}{|l|l|l|l|l|}
\hline Particulars & Megauli & Fulbari & Jutpani & Siddhi \\
\hline Cereals & $5(83.33)$ & $6(100.00)$ & $5(83.33)$ & $6(100.00)$ \\
\hline Oilseeds & $7(87.50)$ & $8(100.00)$ & $4(50.00)$ & $8(100.00)$ \\
\hline Pulses & $10(90.91)$ & $11(100.00)$ & $8(72.73)$ & $9(81.82)$ \\
\hline Vegetables & $24(96.00)$ & $24(96.00)$ & $24(96.00)$ & $23(92.00)$ \\
\hline Fruits & $19(90.48)$ & $21(100.00)$ & $17(80.95)$ & $18(85.71)$ \\
\hline Ornamentals & $23(88.46)$ & $26(100.00)$ & $24(92.31)$ & $15(57.69)$ \\
\hline Wild plants & $154(99.35)$ & $94(60.65)$ & $139(89.68)$ & $143(92.26)$ \\
\hline Total & $242(96.03)$ & $190(75.40)$ & $221(87.70)$ & $222(88.10)$ \\
\hline
\end{tabular}

Source: Survey, 2012

Parenthesis indicate percentages

Observation shows that Megauli has the richest bee flora (242 species), Siddhi (222 species), followed by Jutpani (221 species), and Fulbari (190 species), respectively (Table 2). The bee flora diversity was higher in the Megauli, iddhi and Jutpani VDCs compared to Fulbari VDC (217 species), mainly due to the dominancy of wild flora there. The beekeeping enterprises flourished 
there because of availability of greater diversity of wild bee forage resources for the honeybees. The bee forage plants available in different months with their sources is shown in Table 2 .

Table 2: Bee floral species with pollen/nectar sources with their flowering time of the study areas in Chitwan

\begin{tabular}{|c|c|c|c|c|c|c|c|c|c|c|c|c|c|c|c|}
\hline \multirow{2}{*}{$\begin{array}{l}\text { Common } \\
\text { Name }\end{array}$} & \multirow{2}{*}{$\begin{array}{l}\text { Scientific } \\
\text { Name }\end{array}$} & \multirow[t]{2}{*}{ Family } & \multirow{2}{*}{$\begin{array}{l}\text { Sour } \\
\text { ce }\end{array}$} & \multicolumn{12}{|c|}{ Flowering time } \\
\hline & & & & $\mathbf{J}$ & $\mathbf{F}$ & $\mathbf{M}$ & $\mathbf{A}$ & $\mathbf{M}$ & \begin{tabular}{|l|} 
Ju \\
n
\end{tabular} & $\mathbf{J}$ & $\mathbf{A}$ & $\mathbf{S}$ & $\mathbf{O}$ & $\mathbf{N}$ & $\mathbf{D}$ \\
\hline \multicolumn{16}{|l|}{ Cereal } \\
\hline Rice & $\begin{array}{l}\text { Oryza sativa } \\
\text { L. }\end{array}$ & Poaceae & $\mathrm{P} 2$ & & & & & $\sqrt{ }$ & $\sqrt{ }$ & & & $\sqrt{ }$ & $\sqrt{ }$ & & \\
\hline Maize & Zea mays L. & Poaceae & $\mathrm{P} 1$ & $\sqrt{ }$ & & & & & $\sqrt{ }$ & $\sqrt{ }$ & $\sqrt{ }$ & $\sqrt{ }$ & $\sqrt{ }$ & $\sqrt{ }$ & \\
\hline $\begin{array}{l}\text { Finger } \\
\text { millet }\end{array}$ & $\begin{array}{l}\text { Eleusine } \\
\text { coracana }(\mathrm{L} .) \\
\text { Geartn. }\end{array}$ & Poaceae & $\mathrm{P} 2$ & & & $\sqrt{ }$ & $\sqrt{ }$ & & & & & & & & \\
\hline Wheat & $\begin{array}{l}\text { Triticum } \\
\text { aestivum } \mathrm{L} .\end{array}$ & Poaceae & $\mathrm{P} 2$ & $\sqrt{ }$ & $\sqrt{ }$ & $\sqrt{ }$ & & & & & & & & & \\
\hline Barley & $\begin{array}{l}\text { Avena } \\
\text { sativum } \mathrm{L} .\end{array}$ & Poaceae & $\mathrm{P} 2$ & & $\sqrt{ }$ & $\sqrt{ }$ & & & & & & & & & \\
\hline Buckwheat & $\begin{array}{l}\text { Fagopyrum } \\
\text { esculentum } \\
\text { Moench }\end{array}$ & Polygonaceae & N1P1 & $\sqrt{ }$ & $\sqrt{ }$ & $\sqrt{ }$ & & & & & & & $\sqrt{ }$ & $\sqrt{ }$ & $\sqrt{ }$ \\
\hline \multicolumn{16}{|c|}{ Pulse crops } \\
\hline Pigeon pea & $\begin{array}{l}\text { Cajanus } \\
\text { cajan } \\
\text { Millsp. }\end{array}$ & Fabaceae & $\mathrm{P} 2$ & $\sqrt{ }$ & $\sqrt{ }$ & & & & & & & & & & \\
\hline Lentil & $\begin{array}{l}\text { Lens } \\
\text { culinaris } \\
\text { Medikus }\end{array}$ & Fabaceae & $\mathrm{N} 2$ & & & & & & & & & & & $\sqrt{ }$ & $\sqrt{ }$ \\
\hline Chickpea & $\begin{array}{l}\text { Cicer } \\
\text { arietinum } \mathrm{L} .\end{array}$ & Fabaceae & $\mathrm{N} 2 \mathrm{P} 2$ & & $\sqrt{ }$ & $\sqrt{ }$ & & & & & & & & & \\
\hline Black gram & $\begin{array}{l}\begin{array}{l}\text { Phaseolus } \\
\text { mungo (L.) } \\
\text { Hepper }\end{array} \\
\end{array}$ & Fabaceae & N1 & & & & & & & & $\sqrt{ }$ & $\sqrt{ }$ & $\sqrt{ }$ & & \\
\hline Green gram & $\begin{array}{l}\text { Phaseolus } \\
\text { radiates } \mathrm{L} .\end{array}$ & Fabaceae & N1P1 & & & & & & & & $\sqrt{ }$ & & & & \\
\hline Peas & $\begin{array}{l}\text { Pisum } \\
\text { sativum } \mathrm{L} .\end{array}$ & Fabaceae & $\mathrm{P} 2$ & & & $\sqrt{ }$ & & & & & $\sqrt{ }$ & $\sqrt{ }$ & & & \\
\hline Cowpea & $\begin{array}{l}\text { Vigna } \\
\text { unguiculata } \\
\text { (L.) Walp. }\end{array}$ & Fabaceae & $\mathrm{P} 2$ & & & & & $\sqrt{ }$ & $\sqrt{ }$ & $\sqrt{ }$ & $\sqrt{ }$ & $\sqrt{ }$ & & & \\
\hline Soybean & $\begin{array}{l}\text { Glysine max } \\
\text { (L.) Merr. }\end{array}$ & Fabaceae & $\mathrm{N} 2 \mathrm{P} 2$ & & & & & & & & $\sqrt{ }$ & $\sqrt{ }$ & & & \\
\hline Lima beans & $\begin{array}{l}\text { Phaseolus } \\
\text { lunatus L. }\end{array}$ & Fabaceae & $\mathrm{N} 2$ & & & & & & & & & & $\sqrt{ }$ & $\sqrt{ }$ & \\
\hline Rice beans & $\begin{array}{l}\text { Vigna } \\
\text { umbellate L. }\end{array}$ & Fabaceae & $\mathrm{P} 2$ & & & & & $\sqrt{ }$ & $\sqrt{ }$ & & & & & & \\
\hline $\begin{array}{l}\text { Kidneybean } \\
\text { (Ghiu simi) }\end{array}$ & $\begin{array}{l}\text { Phaseolus } \\
\text { vulgaris } \mathrm{L} .\end{array}$ & Fabaceae & $\mathrm{N} 2$ & & & & & & & & & & $\sqrt{ }$ & $\sqrt{ }$ & \\
\hline
\end{tabular}




\begin{tabular}{|c|c|c|c|c|c|c|c|c|c|c|c|c|c|c|c|}
\hline \multicolumn{16}{|c|}{ Oilseed crops } \\
\hline Rapeseed & $\begin{array}{l}\text { Brassica } \\
\text { campestris } \\
\text { var toria } \mathrm{L} .\end{array}$ & Brassicaceae & N1P1 & $\sqrt{ }$ & $\sqrt{ }$ & & & & & & & & & & $\sqrt{ }$ \\
\hline Sarson & $\begin{array}{l}\text { Brassica } \\
\text { campestris } \\
\text { var. sarson } \\
\text { Prain }\end{array}$ & Brassicaceae & N1P1 & $\sqrt{ }$ & $\sqrt{ }$ & & & & & & & & & & $\sqrt{ }$ \\
\hline Rayo & $\begin{array}{l}\text { Brassica } \\
\text { juncea subsp } \\
\text { rugosa } \\
\text { Roxb.) Prain }\end{array}$ & Brassicaceae & N1P1 & & $\sqrt{ }$ & $\sqrt{ }$ & $\sqrt{ }$ & & & & & & & & \\
\hline $\begin{array}{l}\text { Black } \\
\text { mustard }\end{array}$ & $\begin{array}{l}\text { Brassica } \\
\text { nigra } \mathrm{L} .\end{array}$ & Brassicaceae & $\mathrm{N} 2 \mathrm{P} 2$ & & & & & & & & & $\sqrt{ }$ & $\sqrt{ }$ & & \\
\hline Niger & $\begin{array}{l}\text { Guizotia } \\
\text { abyssinica } \\
\text { (L.f.) Cass. }\end{array}$ & Compositae & N1P1 & & & & & & & $\sqrt{ }$ & $\sqrt{ }$ & $\sqrt{ }$ & $\sqrt{ }$ & & \\
\hline Sunflower & $\begin{array}{l}\text { Helianthus } \\
\text { annuus L. }\end{array}$ & Compositae & N1P1 & & & & & & & $\sqrt{ }$ & $\sqrt{ }$ & $\sqrt{ }$ & & & \\
\hline Linseed & $\begin{array}{l}\text { Linum } \\
\text { usitatissimum } \\
\text { L. }\end{array}$ & Linaceae & $\mathrm{N} 2 \mathrm{P} 2$ & & $\sqrt{ }$ & $\sqrt{ }$ & & & & & & & & & \\
\hline Sesame & $\begin{array}{l}\text { Sesamum } \\
\text { orientale L. }\end{array}$ & Pedaliaceae & N1P1 & & & & $\sqrt{ }$ & $\sqrt{ }$ & $\sqrt{ }$ & $\sqrt{ }$ & & & & & \\
\hline \multicolumn{16}{|c|}{ Vegetable crops } \\
\hline Amaranths & $\begin{array}{l}\text { Amaranthus } \\
\text { hybridus } \\
\text { subsp. } \\
\text { cruantus (L.) } \\
\text { Thell. }\end{array}$ & $\begin{array}{l}\text { Amaranthacea } \\
\mathrm{e}\end{array}$ & N2P2 & & & & & & & $\sqrt{ }$ & $\sqrt{ }$ & & & & \\
\hline $\begin{array}{l}\text { Lady's } \\
\text { finger }\end{array}$ & $\begin{array}{l}\text { Abelmoschus } \\
\text { esculentus } \\
\text { (L.) Moench }\end{array}$ & Malvaceae & N2P2 & & & $\sqrt{ }$ & $\sqrt{ }$ & $\sqrt{ }$ & $\sqrt{ }$ & $\sqrt{ }$ & $\sqrt{ }$ & & & & \\
\hline Chilli & $\begin{array}{l}\text { Capsicum } \\
\text { annum } \mathrm{L} .\end{array}$ & Solanaceae & $\mathrm{P} 1$ & & & & $\sqrt{ }$ & $\sqrt{ }$ & & & & & & & \\
\hline Capcicum & $\begin{array}{l}\text { Capsicum } \\
\text { frutescens } \mathrm{L} .\end{array}$ & Solanaceae & $\mathrm{P} 1$ & & $\sqrt{ }$ & $\sqrt{ }$ & & & & & & $\sqrt{ }$ & $\sqrt{ }$ & & \\
\hline $\begin{array}{l}\text { Sweet } \\
\text { melon }\end{array}$ & $\begin{array}{l}\text { Cucumis } \\
\text { melo L. }\end{array}$ & Cucurbitaceae & N2P2 & & & & $\sqrt{ }$ & $\sqrt{ }$ & $\sqrt{ }$ & & & & & & \\
\hline Cucumber & $\begin{array}{l}\text { Cucumis } \\
\text { sativus L. }\end{array}$ & Cucurbitaceae & $\mathrm{N} 2 \mathrm{P} 2$ & & & $\sqrt{ }$ & $\sqrt{ }$ & $\sqrt{ }$ & $\sqrt{ }$ & $\sqrt{ }$ & $\sqrt{ }$ & $\sqrt{ }$ & $\sqrt{ }$ & & \\
\hline Coriander & $\begin{array}{l}\text { Coriandrum } \\
\text { sativum } \mathrm{L} \text {. }\end{array}$ & Apiaceae & N1P1 & & $\sqrt{ }$ & $\sqrt{ }$ & $\sqrt{ }$ & & & & & & & & \\
\hline Squash & $\begin{array}{l}\text { Cucurbita } \\
\text { pepo } \\
\text { Duchesne }\end{array}$ & Cucurbitaceae & $\mathrm{P} 2$ & & & $\sqrt{ }$ & $\sqrt{ }$ & & & & & & & & \\
\hline Pumpkin & $\begin{array}{l}\text { Cucurbita } \\
\text { moschata } \\
\text { Duchesne }\end{array}$ & Cucurbitaceae & $\mathrm{P} 1$ & & & & & & & & & & & & \\
\hline
\end{tabular}




\begin{tabular}{|c|c|c|c|c|c|c|c|c|c|c|c|c|c|c|c|}
\hline Cluster bean & $\begin{array}{l}\text { Cyamopsis } \\
\text { tetragonolob } \\
a \text { (L.) Taub. }\end{array}$ & Fabaceae & N2 & $\sqrt{ }$ & $\sqrt{ }$ & $\sqrt{ }$ & & & & & & & $\sqrt{ }$ & $\sqrt{ }$ & $\sqrt{ }$ \\
\hline Bottle gourd & $\begin{array}{l}\text { Lagenaria } \\
\text { siceraria } \\
\text { (Molina) } \\
\text { Standl. }\end{array}$ & Cucurbitaceae & $\mathrm{P} 2$ & & & & & $\sqrt{ }$ & $\sqrt{ }$ & $\sqrt{ }$ & & & & & \\
\hline Ridge gourd & $\begin{array}{l}\text { Luffa } \\
\text { acutangula } \\
\text { (L.) Roxb. }\end{array}$ & Cucurbitaceae & $\mathrm{P} 2$ & & & & & $\sqrt{ }$ & $\sqrt{ }$ & $\sqrt{ }$ & & & & $\sqrt{ }$ & $\sqrt{ }$ \\
\hline $\begin{array}{l}\text { Sponge } \\
\text { gourd }\end{array}$ & $\begin{array}{l}\text { Luffa } \\
\text { aegyptiaca } \\
\text { Mill. }\end{array}$ & Cucurbitaceae & P1 & & & & & $\sqrt{ }$ & $\sqrt{ }$ & $\sqrt{ }$ & $\sqrt{ }$ & $\sqrt{ }$ & & & \\
\hline Snake gourd & $\begin{array}{l}\text { Trichosanthe } \\
\text { s anguina L. }\end{array}$ & Cucurbitaceae & $\mathrm{P} 2$ & & & & & $\sqrt{ }$ & $\sqrt{ }$ & $\sqrt{ }$ & $\sqrt{ }$ & & & & \\
\hline Tomato & $\begin{array}{l}\text { Lycopersicon } \\
\text { esculentum } \\
\text { Mill. }\end{array}$ & Solanaceae & P1 & $\sqrt{ }$ & $\sqrt{ }$ & $\sqrt{ }$ & $\sqrt{ }$ & & & & & $\sqrt{ }$ & $\sqrt{ }$ & $\sqrt{ }$ & $\sqrt{ }$ \\
\hline Bitter gourd & $\begin{array}{l}\text { Momordica } \\
\text { charantia } \mathrm{L} .\end{array}$ & Cucurbitaceae & $\mathrm{P} 2$ & & & & $\sqrt{ }$ & $\sqrt{ }$ & $\sqrt{ }$ & $\sqrt{ }$ & $\sqrt{ }$ & & & & \\
\hline Radish & $\begin{array}{l}\text { Raphanus } \\
\text { sativus L. }\end{array}$ & Brassicaceae & N1P1 & & $\sqrt{ }$ & $\sqrt{ }$ & $\sqrt{ }$ & & & & & & & & \\
\hline Brinjal & $\begin{array}{l}\text { Solanum } \\
\text { melongena L. }\end{array}$ & Solanaceae & $\mathrm{N} 2 \mathrm{P} 2$ & & $\sqrt{ }$ & $\sqrt{ }$ & $\sqrt{ }$ & $\sqrt{ }$ & $\sqrt{ }$ & $\sqrt{ }$ & $\sqrt{ }$ & $\sqrt{ }$ & $\sqrt{ }$ & & \\
\hline Musk gourd & $\begin{array}{l}\text { Benincasa } \\
\text { hispida } \\
\text { (Thunb.) } \\
\text { Cogn. }\end{array}$ & $\begin{array}{l}\text { Cucurbitacaea } \\
\text { e }\end{array}$ & $\mathrm{N} 2 \mathrm{P} 2$ & & & & & & $\sqrt{ }$ & $\sqrt{ }$ & $\sqrt{ }$ & $\sqrt{ }$ & $\sqrt{ }$ & & \\
\hline Broccoli & $\begin{array}{l}\text { Brassica } \\
\text { oleracea var. } \\
\text { italica } \text { Plenck }\end{array}$ & Brassicaceae & N1P1 & & & $\sqrt{ }$ & $\sqrt{ }$ & & & & & & & & \\
\hline $\begin{array}{l}\text { Sweet } \\
\text { melon }\end{array}$ & $\begin{array}{l}\text { Citrullus } \\
\text { vulgaris } \\
\text { Schrad } \\
\end{array}$ & Cucurbitaceae & $\mathrm{N} 2 \mathrm{P} 2$ & & & $\sqrt{ }$ & $\sqrt{ }$ & & & & & & & & \\
\hline Spinach & $\begin{array}{l}\text { Lepidium } \\
\text { sativum } \mathrm{L} .\end{array}$ & Brassicaceae & $\mathrm{N} 2$ & $\sqrt{ }$ & $\sqrt{ }$ & & & & & & & & & & \\
\hline Lettuce & $\begin{array}{l}\text { Spinacea } \\
\text { oleracea } \mathrm{L} .\end{array}$ & $\begin{array}{l}\text { Chenopodiace } \\
\text { ae }\end{array}$ & $\mathrm{P} 2$ & & $\sqrt{ }$ & $\sqrt{ }$ & & & & & & & & & \\
\hline Fenugreek & $\begin{array}{l}\text { Trigonella } \\
\text { foenum } \\
\text { graecum L. }\end{array}$ & Leguminosae & $\mathrm{N} 2 \mathrm{P} 2$ & & $\sqrt{ }$ & $\sqrt{ }$ & $\sqrt{ }$ & & & & & & & & \\
\hline Fababeans & Vicia feba L. & Leguminosae & $\mathrm{N} 2 \mathrm{P} 2$ & $\sqrt{ }$ & $\sqrt{ }$ & $\sqrt{ }$ & & & & & & & & & \\
\hline \multicolumn{16}{|l|}{ Fruit crops } \\
\hline $\begin{array}{l}\text { Custard } \\
\text { apple }\end{array}$ & $\begin{array}{l}\text { Annona } \\
\text { squamosa } \mathrm{L} .\end{array}$ & Annonoceae & N1P2 & & & & $\sqrt{ }$ & $\sqrt{ }$ & $\sqrt{ }$ & & & & & & \\
\hline Jack fruit & $\begin{array}{l}\text { Artocarpus } \\
\text { integer } \\
\text { (Thunb.) } \\
\text { Merr }\end{array}$ & Moraceae & $\mathrm{P} 2$ & & $\sqrt{ }$ & $\sqrt{ }$ & & & & & & & & & \\
\hline
\end{tabular}


[Rijal et. al., Vol.6 (Iss.11): November 2018]

(Received: October 23, 2018 - Accepted: November 25, 2018)

DOI: 10.5281/zenodo.1928975

\begin{tabular}{|c|c|c|c|c|c|c|c|c|c|c|c|c|c|c|c|}
\hline Papaya & $\begin{array}{l}\text { Carica } \\
\text { papaya } \mathrm{L} .\end{array}$ & Caricaceae & $\mathrm{N} 2 \mathrm{P} 2$ & & & & $\sqrt{ }$ & $\sqrt{ }$ & $\sqrt{ }$ & & & & & & \\
\hline Lime & $\begin{array}{l}\text { Citrus } \\
\text { aurantifolia } \\
\text { (Christm.) } \\
\text { Swingle }\end{array}$ & Rutaceae & N1P1 & & $\sqrt{ }$ & $\sqrt{ }$ & & & & & & & & & \\
\hline Pumelo & $\begin{array}{l}\text { Citrus } \\
\text { grandis (L.) } \\
\text { Osbeck }\end{array}$ & Rutaceae & N1P1 & & & $\sqrt{ }$ & $\sqrt{ }$ & & & & & & & & \\
\hline $\begin{array}{l}\text { Rough } \\
\text { lemon }\end{array}$ & $\begin{array}{l}\text { Citrus } \\
\text { jambhiri } \\
\text { Lush. }\end{array}$ & Rutaceae & N1P1 & & & $\sqrt{ }$ & $\sqrt{ }$ & & & & & & & & \\
\hline $\begin{array}{l}\text { Indian } \\
\text { sweet lime }\end{array}$ & $\begin{array}{l}\text { Citrus } \\
\text { limetttoides } \\
\text { Tanaka }\end{array}$ & Rutaceae & N1P1 & & & $\sqrt{ }$ & $\sqrt{ }$ & & & & & & & & \\
\hline Lemon & $\begin{array}{l}\text { Citrus limon } \\
\text { (L.) Osbeck }\end{array}$ & Rutaceae & N1P1 & & & $\sqrt{ }$ & $\sqrt{ }$ & & & & & & & & \\
\hline Citron & $\begin{array}{l}\text { Citrus medica } \\
\text { L. }\end{array}$ & Rutaceae & N1P1 & & & $\sqrt{ }$ & $\sqrt{ }$ & & & & & & & & \\
\hline Grapefruit & $\begin{array}{l}\text { Citrus } \\
\text { paradisi } \\
\text { Macfad. }\end{array}$ & Rutaceae & N1P1 & & & $\sqrt{ }$ & $\sqrt{ }$ & & & & & & & & \\
\hline Orange & $\begin{array}{l}\text { Citrus } \\
\text { reticulata } \\
\text { Blanco }\end{array}$ & Rutaceae & N1P1 & & & $\sqrt{ }$ & $\sqrt{ }$ & & & & & & & & \\
\hline Lichi & $\begin{array}{l}\text { Litchi } \\
\text { chinensis } \\
\text { Sonner }\end{array}$ & Sapindaceae & N1P1 & & & $\sqrt{ }$ & $\sqrt{ }$ & & & & & & & & \\
\hline Mango & $\begin{array}{l}\text { Mangifera } \\
\text { indica } \mathrm{L} \text {. }\end{array}$ & $\begin{array}{l}\text { Anacardiacea } \\
\mathrm{e}\end{array}$ & N2P1 & & & $\sqrt{ }$ & $\sqrt{ }$ & & & & & & & & \\
\hline Banana & $\begin{array}{l}\text { Musa } \\
\text { paradisiaca } \\
\text { L. }\end{array}$ & Musaceae & $\mathrm{N} 2 \mathrm{P} 2$ & $\sqrt{ }$ & $\sqrt{ }$ & $\sqrt{ }$ & $\sqrt{ }$ & $\sqrt{ }$ & $\sqrt{ }$ & & & & & & \\
\hline Guava & $\begin{array}{l}\text { Psidium } \\
\text { guajava L. }\end{array}$ & Myrtaceae & $\mathrm{P} 2$ & & & $\sqrt{ }$ & $\sqrt{ }$ & $\sqrt{ }$ & & & & & & & \\
\hline Pear & $\begin{array}{l}\text { Pyrus } \\
\text { communis L. }\end{array}$ & Rosaceae & N1P1 & & & & & & & & & & & & \\
\hline $\begin{array}{l}\text { Pomegranat } \\
\mathrm{e}\end{array}$ & $\begin{array}{l}\text { Punica } \\
\text { granatum L. }\end{array}$ & Lythraceae & N2P1 & & & & $\sqrt{ }$ & $\sqrt{ }$ & & & & & & & \\
\hline Peach & $\begin{array}{l}\text { Prunus } \\
\text { persica (L.) } \\
\text { Batsch }\end{array}$ & Rosaceae & N1P1 & & $\sqrt{ }$ & $\sqrt{ }$ & & & & & & & & & \\
\hline Areca nut & $\begin{array}{l}\text { Annona } \\
\text { reticulata L. }\end{array}$ & Annonaceae & $\mathrm{N} 2 \mathrm{P} 2$ & & & & & $\sqrt{ }$ & & & & & & & \\
\hline Coconut & $\begin{array}{l}\text { Cocos } \\
\text { nucifera } \mathrm{L} .\end{array}$ & Arecaceae & $\mathrm{N} 2$ & $\sqrt{ }$ & $\sqrt{ }$ & $\sqrt{ }$ & $\sqrt{ }$ & $\sqrt{ }$ & $\sqrt{ }$ & $\sqrt{ }$ & $\sqrt{ }$ & $\sqrt{ }$ & $\sqrt{ }$ & $\sqrt{ }$ & $\sqrt{ }$ \\
\hline Persimon & $\begin{array}{l}\text { Diospyros } \\
\text { virginiana } \\
\text { L.Physalis } \\
\end{array}$ & Ebenaceae & $\mathrm{N} 2 \mathrm{P} 2$ & & & $\sqrt{ }$ & $\sqrt{ }$ & & & & & & & & \\
\hline
\end{tabular}


[Rijal et. al., Vol.6 (Iss.11): November 2018]

(Received: October 23, 2018 - Accepted: November 25, 2018)

DOI: 10.5281/zenodo.1928975

\begin{tabular}{|c|c|c|c|c|c|c|c|c|c|c|c|c|c|c|c|}
\hline $\begin{array}{l}\text { Callistemon } \\
\text { (kalkiful) }\end{array}$ & $\begin{array}{l}\text { Callistemon } \\
\text { citrinus } \\
\text { (Curtis) } \\
\text { Skeels }\end{array}$ & Myrtaceae & N1P1 & & $\sqrt{ }$ & $\sqrt{ }$ & $\sqrt{ }$ & $\sqrt{ }$ & $\sqrt{ }$ & $\sqrt{ }$ & $\sqrt{ }$ & & & & \\
\hline $\begin{array}{l}\text { Crysanthim } \\
\text { um }\end{array}$ & $\begin{array}{l}\text { Chrysanthem } \\
\text { um segetum } \\
\text { L. }\end{array}$ & Asteraceae & $\mathrm{N} 2 \mathrm{P} 2$ & & & & & & & & $\sqrt{ }$ & $\sqrt{ }$ & $\sqrt{ }$ & $\sqrt{ }$ & $\sqrt{ }$ \\
\hline Gulmohar & 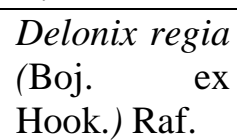 & Fabaceae & $\mathrm{N} 2 \mathrm{P} 2$ & & & & & $\sqrt{ }$ & $\sqrt{ }$ & & & & & & \\
\hline Gomphrena & $\begin{array}{l}\text { Gomphrena } \\
\text { globosa L. }\end{array}$ & $\begin{array}{l}\text { Amaranthacea } \\
\mathrm{e}\end{array}$ & $\mathrm{P} 2$ & $\sqrt{ }$ & & & & & & $\sqrt{ }$ & $\sqrt{ }$ & $\sqrt{ }$ & $\sqrt{ }$ & $\sqrt{ }$ & $\sqrt{ }$ \\
\hline Jasmin & $\begin{array}{l}\text { Jasminium } \\
\text { sambac (L.) } \\
\text { Ait. }\end{array}$ & Oleaceae & $\mathrm{N} 2$ & & & & & & & & & $\sqrt{ }$ & $\sqrt{ }$ & $\sqrt{ }$ & $\sqrt{ }$ \\
\hline Rose & Rosa indica $\mathrm{L}$ & Rosaceae & $\mathrm{N} 2 \mathrm{P} 2$ & & & & & & $\sqrt{ }$ & $\sqrt{ }$ & & & & & \\
\hline Marigold & $\begin{array}{l}\text { Tagetes } \\
\text { patula } \mathrm{L} .\end{array}$ & Compositae & $\mathrm{N} 2 \mathrm{P} 2$ & & & & & & & & & $\sqrt{ }$ & $\sqrt{ }$ & $\sqrt{ }$ & $\sqrt{ }$ \\
\hline Zinnia & $\begin{array}{l}\text { Crassina } \\
\text { elegans } \\
\text { (Jacq.) } \\
\text { Kuntze }\end{array}$ & Asteraceae & $\mathrm{N} 1 \mathrm{P} 2$ & & & & & & $\sqrt{ }$ & $\sqrt{ }$ & $\sqrt{ }$ & $\sqrt{ }$ & $\sqrt{ }$ & & \\
\hline $\begin{array}{l}\text { Chinese } \\
\text { rose }\end{array}$ & $\begin{array}{l}\text { Hibiscus } \\
\text { rosa-sinensis } \\
\text { L. }\end{array}$ & Malvaceae & $\mathrm{N} 2 \mathrm{P} 2$ & & & $\sqrt{ }$ & $\sqrt{ }$ & $\sqrt{ }$ & $\sqrt{ }$ & $\sqrt{ }$ & & & & & \\
\hline Bottlebrush & $\begin{array}{ll}\text { Grevillea } & \\
\text { robusta A. } & \text { A. } \\
\text { Cunn. ex } & \text { R. } \\
\text { Br. } & \\
\end{array}$ & Proteaceae & N1P1 & & & & $\sqrt{ }$ & $\sqrt{ }$ & & & & & & & \\
\hline $\begin{array}{l}\text { Night } \\
\text { jasmine }\end{array}$ & $\begin{array}{l}\text { Nyctanthes } \\
\text { arbor-tristis } \\
\text { L. }\end{array}$ & Oleaceae & N1P1 & & & & & $\sqrt{ }$ & $\sqrt{ }$ & $\sqrt{ }$ & $\sqrt{ }$ & $\sqrt{ }$ & $\sqrt{ }$ & & \\
\hline $\begin{array}{l}\text { Garden } \\
\text { balsam }\end{array}$ & $\begin{array}{l}\text { Impatiens } \\
\text { balsamina L. }\end{array}$ & $\begin{array}{l}\text { Balsamininac } \\
\text { eae }\end{array}$ & N1P2 & & & & & & $\sqrt{ }$ & $\sqrt{ }$ & $\sqrt{ }$ & & & & \\
\hline $\begin{array}{l}\text { Wax } \\
\text { mallow }\end{array}$ & $\begin{array}{l}\text { Malvaviscus } \\
\text { arboreus } \\
\text { Cav. }\end{array}$ & Malvaceae & N1P1 & $\sqrt{ }$ & $\sqrt{ }$ & $\sqrt{ }$ & $\sqrt{ }$ & $\sqrt{ }$ & $\sqrt{ }$ & $\sqrt{ }$ & $\sqrt{ }$ & $\sqrt{ }$ & $\sqrt{ }$ & $\sqrt{ }$ & $\sqrt{ }$ \\
\hline Agava & $\begin{array}{l}\text { Agave } \\
\text { americana L. }\end{array}$ & Agavaceae & $\mathrm{N} 2 \mathrm{P} 2$ & & & & & & & & $\sqrt{ }$ & $\sqrt{ }$ & $\sqrt{ }$ & $\sqrt{ }$ & $\sqrt{ }$ \\
\hline Hollyhook & $\begin{array}{l}\text { Althaea rosea } \\
\text { (L.) Cav. }\end{array}$ & Malvaceae & $\mathrm{N} 2 \mathrm{P} 2$ & & & & $\sqrt{ }$ & $\sqrt{ }$ & $\sqrt{ }$ & & & & & & \\
\hline Calendula & $\begin{array}{l}\text { Calendula } \\
\text { offisinalis L. }\end{array}$ & Compositae & $\mathrm{N} 2 \mathrm{P} 2$ & & $\sqrt{ }$ & $\sqrt{ }$ & $\sqrt{ }$ & & & & & & & & \\
\hline Dahlia & $\begin{array}{l}\text { Dahlia } \\
\text { pinnata Cav. }\end{array}$ & Compositeae & $\mathrm{P} 2$ & $\sqrt{ }$ & & & & & & & $\sqrt{ }$ & $\sqrt{ }$ & $\sqrt{ }$ & $\sqrt{ }$ & $\sqrt{ }$ \\
\hline Dianthus & $\begin{array}{l}\text { Dianthus } \\
\text { chinensis L. }\end{array}$ & $\begin{array}{l}\text { Cariyophyllac } \\
\text { eae }\end{array}$ & $\mathrm{P} 2$ & & $\sqrt{ }$ & $\sqrt{ }$ & $\sqrt{ }$ & $\sqrt{ }$ & & & & & & & \\
\hline Euphorbia & $\begin{array}{l}\text { Euphorbia } \\
\text { pulcherrima }\end{array}$ & $\begin{array}{l}\text { Euphorbiacea } \\
\mathrm{e}\end{array}$ & $\mathrm{N} 2 \mathrm{P} 2$ & $\sqrt{ }$ & $\sqrt{ }$ & & & & & & & & & & \\
\hline
\end{tabular}




\begin{tabular}{|c|c|c|c|c|c|c|c|c|c|c|c|c|c|c|c|c|}
\hline & $\begin{array}{l}\text { Willd.ex } \\
\text { Klotzsch. }\end{array}$ & & & & & & & & & & & & & & & \\
\hline Ixora & $\begin{array}{l}\text { Ixora } \\
\text { coccinea } \mathrm{L} .\end{array}$ & Rubiaceae & $\mathrm{N} 2$ & & & & & & & $\sqrt{ }$ & $\sqrt{ }$ & $\sqrt{ }$ & $\sqrt{ }$ & $\sqrt{ }$ & & \\
\hline \begin{tabular}{|l} 
Lagerstromi \\
a
\end{tabular} & $\begin{array}{l}\text { Lagerstromia } \\
\text { indica } \mathrm{L} \text {. }\end{array}$ & Lythraceae & N1P1 & & & & & & & $\sqrt{ }$ & $\sqrt{ }$ & & & & & \\
\hline Poppy & $\begin{array}{l}\text { Papaver } \\
\text { somniferum } \\
\text { L. }\end{array}$ & Papavaraceae & $\mathrm{P} 1$ & $\sqrt{ }$ & $\sqrt{ }$ & & & & & & & & & & & $\sqrt{ }$ \\
\hline $\begin{array}{l}\text { Clove } \\
\text { flower (big) }\end{array}$ & $\begin{array}{l}\text { Russelia } \\
\text { equisetiformi } \\
s \text { Schltdl. \& } \\
\text { Cham. }\end{array}$ & $\begin{array}{l}\text { Schophulariac } \\
\text { eae }\end{array}$ & N1 & & & & & & & $\sqrt{ }$ & $\sqrt{ }$ & $\sqrt{ }$ & & & & \\
\hline $\begin{array}{l}\text { Clove } \\
\text { flower } \\
\text { (small) }\end{array}$ & $\begin{array}{l}\text { Russelia } \\
\text { juncea Zucc. }\end{array}$ & $\begin{array}{l}\text { Plantaginacea } \\
\text { e }\end{array}$ & N1 & & & & & & & $\sqrt{ }$ & $\sqrt{ }$ & $\sqrt{ }$ & & & & \\
\hline Cuphea & $\begin{array}{l}\text { Cuphea } \\
\text { micropetala } \\
\text { Kunth }\end{array}$ & Lythroideae & N1P2 & & & 1 & & & & $\sqrt{ }$ & $\sqrt{ }$ & $\sqrt{ }$ & $\sqrt{ }$ & $\sqrt{ }$ & & \\
\hline \multirow{2}{*}{\multicolumn{17}{|c|}{ Weeds/wild plants }} \\
\hline & & & & & & & & & & & & & & & & \\
\hline Spear mint & $\begin{array}{l}\text { Mentha } \\
\text { spicata } \mathrm{L} \text {. }\end{array}$ & Lamiaceae & $\mathrm{N} 2$ & & & & & & & & $\sqrt{ }$ & $\sqrt{ }$ & & & & \\
\hline Tulasi & $\begin{array}{l}\text { Ocimum } \\
\text { tenuiflorum } \\
\text { L. }\end{array}$ & Lamiaceae & $\mathrm{N} 2$ & & & & & & & & & $\sqrt{ }$ & $\sqrt{ }$ & $\sqrt{ }$ & & \\
\hline $\begin{array}{l}\text { Basil } \\
\text { (Babari) }\end{array}$ & $\begin{array}{l}\text { Ocipum } \\
\text { basilicum L. }\end{array}$ & Lamiaceae & $\mathrm{N} 2$ & $\sqrt{ }$ & $\sqrt{ }$ & & & & & & & & & & & \\
\hline Mugwort & $\begin{array}{l}\text { Artemesia } \\
\text { indica Willd. }\end{array}$ & Asteraceae & $\mathrm{N} 2$ & & & & & & & & & & $\sqrt{ }$ & $\sqrt{ }$ & $\sqrt{ }$ & \\
\hline $\begin{array}{l}\text { Acacia } \\
\text { (khair) }\end{array}$ & $\begin{array}{l}\text { Acacia } \\
\text { catechu (L.f.) } \\
\text { Wilid. }\end{array}$ & Leguminaceae & $\mathrm{N} 2 \mathrm{P} 2$ & & & & & & $\sqrt{ }$ & & & & & & & \\
\hline Neem & $\begin{array}{l}\text { Azadirachta } \\
\text { indica A. } \\
\text { Juss. }\end{array}$ & Meliaceae & $\mathrm{N} 2 \mathrm{P} 2$ & & & & & & $\sqrt{ }$ & & & & & & & \\
\hline $\begin{array}{l}\begin{array}{l}\text { Bombox } \\
\text { (Simal) }\end{array} \\
\end{array}$ & $\begin{array}{l}\text { Bombax } \\
\text { ceiba } \mathrm{L} .\end{array}$ & Bombacaceae & N1P1 & & & 1 & & & & & & & & & & \\
\hline $\begin{array}{l}\text { Chenopodiu } \\
\mathrm{m}\end{array}$ & $\begin{array}{l}\text { Chenopodiu } \\
\text { m album L. }\end{array}$ & $\begin{array}{l}\text { Chenopodiace } \\
\text { ae }\end{array}$ & $\mathrm{P} 2$ & $\sqrt{ }$ & $\sqrt{ }$ & 1 & & & & & & & & & & \\
\hline Sissoo & $\begin{array}{l}\text { Dalbergia } \\
\text { sissoo Roxb. }\end{array}$ & Fabaceae & N1 & & & & & & & & & & & & & \\
\hline Dhatura & $\begin{array}{l}\text { Datura metel } \\
\text { L. }\end{array}$ & Solanaceae & $\mathrm{P} 2$ & $\sqrt{ }$ & $\sqrt{ }$ & 1 & & & & & & & & & & \\
\hline Mulberry & $\begin{array}{l}\text { Morus alba } \\
\text { L. }\end{array}$ & Moraceae & $\mathrm{P} 1$ & & & 1 & & & & & & & & & & \\
\hline
\end{tabular}


[Rijal et. al., Vol.6 (Iss.11): November 2018]

(Received: October 23, 2018 - Accepted: November 25, 2018)

DOI: 10.5281/zenodo.1928975

\begin{tabular}{|c|c|c|c|c|c|c|c|c|c|c|c|c|c|c|c|}
\hline Parthenium & $\begin{array}{l}\text { Parthenium } \\
\text { hysterophoru } \\
s \text { L. }\end{array}$ & Asteraceae & $\mathrm{P} 1$ & & & $\sqrt{ }$ & $\sqrt{ }$ & $\sqrt{ }$ & $\sqrt{ }$ & $\sqrt{ }$ & $\sqrt{ }$ & $\sqrt{ }$ & $\sqrt{ }$ & $\sqrt{ }$ & $\sqrt{ }$ \\
\hline $\begin{array}{l}\text { Phyllanthus } \\
\text { (Amala) }\end{array}$ & $\begin{array}{l}\text { Phyllanthus } \\
\text { emblica L. }\end{array}$ & $\begin{array}{l}\text { Euphorbiacea } \\
\text { e }\end{array}$ & N1 & & & & $\sqrt{ }$ & $\sqrt{ }$ & & & & & & & \\
\hline Toona & $\begin{array}{l}\text { Toona ciliata } \\
\text { M.Roem. }\end{array}$ & Meliaceae & N1P2 & & & & $\sqrt{ }$ & & & & & & & & \\
\hline Wild Ber & $\begin{array}{l}\text { Zizyphus } \\
\text { oxyphylla } \\
\text { Edgew. } \\
\end{array}$ & Rhamnaceae & $\mathrm{N} 2 \mathrm{P} 2$ & & & & & & $\sqrt{ }$ & $\sqrt{ }$ & & & & & \\
\hline Ber & $\begin{array}{l}\text { Ziziphus } \\
\text { mauritiana } \\
\text { Lam. }\end{array}$ & Rhamnaceae & $\mathrm{N} 2 \mathrm{P} 2$ & & & & & & & & & $\sqrt{ }$ & $\sqrt{ }$ & & \\
\hline $\begin{array}{l}\text { Albizia } \\
\text { (Padke) }\end{array}$ & $\begin{array}{l}\text { Albizia } \\
\text { julibrissin } \\
\text { Durazz. }\end{array}$ & Leguminaceae & N1P1 & & & & & & $\sqrt{ }$ & $\sqrt{ }$ & & & & & \\
\hline $\begin{array}{l}\text { Melia } \\
\text { (Bakaino) }\end{array}$ & $\begin{array}{l}\text { Melia } \\
\text { azedarach L. }\end{array}$ & Meliaceae & $\mathrm{P} 2$ & & & & $\sqrt{ }$ & & & & & & & & \\
\hline Premna & $\begin{array}{l}\text { Premna } \\
\text { barbata } \\
\text { (Wall. ex } \\
\text { Schauer) } \\
\text { Kuntze }\end{array}$ & Lamiaceae & $\mathrm{P} 2$ & & & & $\sqrt{ }$ & & & & & & & & \\
\hline $\begin{array}{l}\text { Butternut } \\
\text { tree (Chiuri) }\end{array}$ & $\begin{array}{l}\text { Bassia } \\
\text { butyracea } \\
\text { Roxb. }\end{array}$ & Sapotaceae & N1P1 & $\sqrt{ }$ & $\sqrt{ }$ & & & & & & & & & $\sqrt{ }$ & $\sqrt{ }$ \\
\hline Clestocalyx & $\begin{array}{l}\text { Cleistocalyx } \\
\text { operculatus } \\
\text { (Roxb.) } \\
\text { Merr.\& L.M } \\
\text { Perry } \\
\end{array}$ & Myrtiaceae & N1P1 & & & $\sqrt{ }$ & $\sqrt{ }$ & & & & & & & & \\
\hline Eugenia & $\begin{array}{l}\text { Eugenia } \\
\text { formosa } \\
\text { Wall. }\end{array}$ & Myrtiaceae & N1P1 & & $\sqrt{ }$ & $\sqrt{ }$ & $\sqrt{ }$ & & & & & & & & \\
\hline Syzgium & $\begin{array}{ll}\text { Syzgium } & \\
\text { cumini } & \text { (L.) } \\
\text { Skeels } & \\
\end{array}$ & Myrtiaceae & N1 & & & & $\sqrt{ }$ & $\sqrt{ }$ & $\sqrt{ }$ & & & & & & \\
\hline $\begin{array}{l}\text { Terminalia } \\
\text { (Saj,Asna) }\end{array}$ & $\begin{array}{l}\text { Terminalia } \\
\text { bellirica } \\
\text { (Geartn.) } \\
\text { Roxb. }\end{array}$ & Combretaceae & N1P1 & & & & $\sqrt{ }$ & $\sqrt{ }$ & & & & & & & \\
\hline Calendula & $\begin{array}{l}\text { Cassia fistula } \\
\text { L. }\end{array}$ & Leguminaceae & $\mathrm{N} 2 \mathrm{P} 2$ & & & & & $\sqrt{ }$ & $\sqrt{ }$ & $\sqrt{ }$ & & & & & \\
\hline Sal & $\begin{array}{l}\text { Shorea } \\
\text { robusta } \\
\text { Guartn. }\end{array}$ & $\begin{array}{l}\text { Dipterocarpac } \\
\text { eae }\end{array}$ & $\mathrm{N} 2 \mathrm{P} 2$ & & & $\sqrt{ }$ & $\sqrt{ }$ & & & & & & & & \\
\hline Epil-epil & $\begin{array}{l}\text { Leucaena } \\
\text { tricodes } \\
\text { (Jacq.) Benth }\end{array}$ & Fabaceae & $\mathrm{N} 2 \mathrm{P} 2$ & & & & & & & & & $\sqrt{ }$ & $\sqrt{ }$ & & \\
\hline
\end{tabular}


[Rijal et. al., Vol.6 (Iss.11): November 2018]

(Received: October 23, 2018 - Accepted: November 25, 2018)

DOI: 10.5281/zenodo.1928975

\begin{tabular}{|c|c|c|c|c|c|c|c|c|c|c|c|c|c|c|}
\hline Bird plum & $\begin{array}{l}\text { Berchemia } \\
\text { edgeworthii } \\
\text { Lawson }\end{array}$ & Rhamnaceae & $\mathrm{N} 2 \mathrm{P} 2$ & & & & $\sqrt{ }$ & $\sqrt{ }$ & $\sqrt{ }$ & & & & & \\
\hline Siam weed & $\begin{array}{l}\text { Chromolaena } \\
\text { odorata (L.) } \\
\text { R.M. King \& } \\
\text { H. Rob. }\end{array}$ & Compositae & $\mathrm{P} 2$ & & & & & & & & & & & \\
\hline $\begin{array}{l}\text { Touch-me- } \\
\text { not } \\
\text { (Lajjabati) }\end{array}$ & $\begin{array}{l}\text { Mimosa } \\
\text { pudica } \mathrm{L} .\end{array}$ & Leguminaceae & $\mathrm{P} 1$ & & & & $\sqrt{ }$ & $\sqrt{ }$ & & & & & & \\
\hline $\begin{array}{l}\text { Pogostemon } \\
\text { (Rudilo) }\end{array}$ & $\begin{array}{l}\text { Pogostemon } \\
\text { glaber Benth. }\end{array}$ & Lamiaceae & N1P1 & $\sqrt{ }$ & $\sqrt{ }$ & $\sqrt{ }$ & $\sqrt{ }$ & $\sqrt{ }$ & $\sqrt{ }$ & & & & & \\
\hline $\begin{array}{l}\text { Callicarpa } \\
\text { (Dahikamal } \\
\text { a) }\end{array}$ & $\begin{array}{l}\text { Callicarpa } \\
\text { macrophylla } \\
\text { Val. }\end{array}$ & Rubiaceae & $\mathrm{P} 2$ & & & & & & $\sqrt{ }$ & $\sqrt{ }$ & $\sqrt{ }$ & $\sqrt{ }$ & 1 & \\
\hline $\begin{array}{l}\text { Ipomoea } \\
\text { (Besarum) }\end{array}$ & 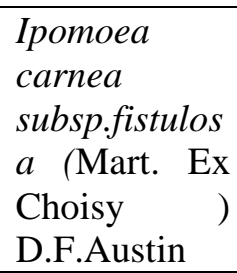 & $\begin{array}{l}\text { Convolvulace } \\
\text { ae }\end{array}$ & $\mathrm{P} 2$ & & & & $\sqrt{ }$ & $\sqrt{ }$ & $\sqrt{ }$ & $\sqrt{ }$ & $\sqrt{ }$ & $\sqrt{ }$ & & \\
\hline Ipomoea & $\begin{array}{l}\text { Ipomoea } \\
\text { aquatica } \\
\text { Forssk. }\end{array}$ & $\begin{array}{l}\text { Convolvulace } \\
\text { ae }\end{array}$ & $\mathrm{P} 2$ & & & & & & & $\sqrt{ }$ & $\sqrt{ }$ & $\sqrt{ }$ & & \\
\hline Bindweed & $\begin{array}{l}\text { Convolvulus } \\
\text { arvensis } \mathrm{L} .\end{array}$ & $\begin{array}{l}\text { Convolvulace } \\
\text { ae }\end{array}$ & $\mathrm{N} 2 \mathrm{P} 2$ & & & & & & & & & & & \\
\hline Knotweed & $\begin{array}{l}\text { Polygonum } \\
\text { spp }\end{array}$ & Poligonaceae & $\mathrm{N} 2 \mathrm{P} 2$ & & & & & $\sqrt{ }$ & $\sqrt{ }$ & $\sqrt{ }$ & $\sqrt{ }$ & $\sqrt{ }$ & & \\
\hline $\begin{array}{l}\text { Malaysian } \\
\text { wild vine } \\
\text { (Simali) }\end{array}$ & $\begin{array}{l}\text { Cissus repens } \\
\text { Lam. }\end{array}$ & Vitaceae & N1P2 & & & & & $\sqrt{ }$ & $\sqrt{ }$ & $\sqrt{ }$ & $\sqrt{ }$ & $\sqrt{ }$ & 1 & \\
\hline $\begin{array}{l}\text { Bermudagra } \\
\text { ss (Dubo) }\end{array}$ & $\begin{array}{l}\text { Cynodon } \\
\text { dactylon (L.) } \\
\text { Pers. }\end{array}$ & Poaceae & $\mathrm{N} 2 \mathrm{P} 2$ & & & & & $\sqrt{ }$ & $\sqrt{ }$ & & & & & \\
\hline $\begin{array}{l}\text { Cassia } \\
\text { (tapre) }\end{array}$ & $\begin{array}{l}\text { Cassia tora } \\
\text { L. }\end{array}$ & Leguminaceae & $\mathrm{N} 2 \mathrm{P} 2$ & & & & & & $\sqrt{ }$ & $\sqrt{ }$ & $\sqrt{ }$ & $\sqrt{ }$ & 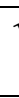 & \\
\hline $\begin{array}{l}\text { Oxalis } \\
\text { (chariamilo) }\end{array}$ & $\begin{array}{l}\text { Oxalis } \\
\text { acetosella } \mathrm{L} .\end{array}$ & Oxalidaceae & $\mathrm{N} 2$ & & $\sqrt{ }$ & $\sqrt{ }$ & $\sqrt{ }$ & & & & & & & \\
\hline Crabgrass & $\begin{array}{l}\text { Digitaria } \\
\text { cilliaris } \\
\text { (Retz.) } \\
\text { Koeler }\end{array}$ & Oleraceae & $\mathrm{N}$ & & & & & & & & & & & \\
\hline $\begin{array}{l}\text { Leucas } \\
\text { (gumpate) }\end{array}$ & $\begin{array}{l}\text { Leucas lanata } \\
\text { Benth. }\end{array}$ & Lamiaceae & N1P1 & $\sqrt{ }$ & $\sqrt{ }$ & $\sqrt{ }$ & $\sqrt{ }$ & & & & & & & \\
\hline Clocasia & $\begin{array}{l}\text { Colocasia } \\
\text { esculenta (L.) } \\
\text { Schott }\end{array}$ & Araceae & $\mathrm{P} 1$ & & & & & & & $\sqrt{ }$ & $\sqrt{ }$ & & & \\
\hline Perilla & $\begin{array}{l}\text { Perilla } \\
\text { frutescens } \\
\text { (L.) Brit. }\end{array}$ & Labiatae & N1P1 & & & & & & & & & & 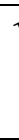 & $\sqrt{ }$ \\
\hline
\end{tabular}


[Rijal et. al., Vol.6 (Iss.11): November 2018]

\begin{tabular}{|c|c|c|c|c|c|c|c|c|c|c|c|c|c|}
\hline $\begin{array}{l}\text { Eupatorium } \\
\text { (Banmara) }\end{array}$ & $\begin{array}{l}\text { Eupatorium } \\
\text { adenophorum } \\
\text { Spreng. }\end{array}$ & Compositae & N1P1 & $\sqrt{ }$ & $\sqrt{ }$ & & & & & & & & \\
\hline $\begin{array}{l}\text { Eupatorium } \\
\text { (Banmara) }\end{array}$ & $\begin{array}{l}\text { Eupatorium } \\
\text { odoratum } \mathrm{L} \text {. }\end{array}$ & Compositae & $\mathrm{N} 2 \mathrm{P} 2$ & & $\sqrt{ }$ & $\sqrt{ }$ & $\sqrt{ }$ & $\sqrt{ }$ & $\sqrt{ }$ & & & & \\
\hline Justicia & $\begin{array}{l}\text { Justicia } \\
\text { adhatoda } \mathrm{L} .\end{array}$ & Acanthaceae & N1P1 & & $\sqrt{ }$ & $\sqrt{ }$ & & & & & & & \\
\hline $\begin{array}{l}\text { Black } \\
\text { Justiicia }\end{array}$ & $\begin{array}{l}\text { Justicia } \\
\text { gendarussa } \\
\text { Burm.f. }\end{array}$ & Acanthaceae & N1 & $\sqrt{ }$ & $\sqrt{ }$ & $\sqrt{ }$ & & & & & & & \\
\hline $\begin{array}{l}\text { Adina } \\
\text { (Karma) }\end{array}$ & $\begin{array}{l}\text { Adina } \\
\text { cordifolia } \\
\text { (Roxb.) } \\
\text { Brandis. }\end{array}$ & Rubiaceae & N1P1 & & & & $\sqrt{ }$ & $\sqrt{ }$ & & & & & \\
\hline Asculus & $\begin{array}{l}\text { Asculus } \\
\text { indica (Wall. } \\
\text { ex Camb.) } \\
\text { Hook. }\end{array}$ & $\begin{array}{l}\text { Hipocastanace } \\
\text { ae }\end{array}$ & N1P1 & & & $\sqrt{ }$ & $\sqrt{ }$ & & & & & & \\
\hline $\begin{array}{l}\text { Ageratum } \\
\text { (Gandhe } \\
\text { jhar) }\end{array}$ & $\begin{array}{l}\text { Ageratum } \\
\text { conizoides L. }\end{array}$ & Compositae & $\mathrm{N} 2 \mathrm{P} 2$ & & & $\sqrt{ }$ & $\sqrt{ }$ & & & & & & \\
\hline Amaranths & $\begin{array}{l}\text { Amaranthus } \\
\text { spinosus L. }\end{array}$ & $\begin{array}{l}\text { Amaranthacea } \\
\mathrm{e}\end{array}$ & $\mathrm{N} 2 \mathrm{P} 2$ & & & & $\sqrt{ }$ & $\sqrt{ }$ & $\sqrt{ }$ & & & & \\
\hline $\begin{array}{l}\text { Anthocepha } \\
\text { lus (Kadam) }\end{array}$ & $\begin{array}{l}\text { Anthocephalu } \\
\text { s chinensis } \\
\text { (Lam.) } \\
\text { A.Rich.ex.W } \\
\text { alp. }\end{array}$ & Rubiaceae & $\mathrm{N} 2 \mathrm{P} 1$ & & & & & & & & & $\sqrt{ }$ & $\sqrt{ }$ \\
\hline $\begin{array}{l}\text { Zeranium } \\
\text { (Tanki) }\end{array}$ & $\begin{array}{l}\text { Bauhinia } \\
\text { purpurea L. }\end{array}$ & Leguminaceae & N1P1 & & & & & & & $\sqrt{ }$ & 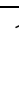 & & \\
\hline Bahunia & $\begin{array}{l}\text { Bahunia } \\
\text { vahlii Wight } \\
\text { \& Arn. }\end{array}$ & Leguminaceae & $\mathrm{N} 2 \mathrm{P} 1$ & & & $\sqrt{ }$ & $\sqrt{ }$ & & & & & & \\
\hline $\begin{array}{l}\text { Orchid tree } \\
\text { (Koilalo) }\end{array}$ & $\begin{array}{l}\text { Bahunia } \\
\text { variegata } \mathrm{L} .\end{array}$ & Leguminaceae & N1P1 & $\sqrt{ }$ & $\sqrt{ }$ & & & & & & & & \\
\hline Bidens & $\begin{array}{l}\text { Bidens pilosa } \\
\text { L. }\end{array}$ & Compositae & $\mathrm{P} 2$ & & & & $\sqrt{ }$ & $\sqrt{ }$ & $\sqrt{ }$ & & & & \\
\hline Breea & $\begin{array}{l}\text { Breea } \\
\text { arvensis (L.) } \\
\text { Less. }\end{array}$ & Compositae & $\mathrm{N} 2 \mathrm{P} 2$ & & $\sqrt{ }$ & $\sqrt{ }$ & $\sqrt{ }$ & & & & & & \\
\hline Casalpinia & $\begin{array}{l}\text { Casalpinia } \\
\text { decapetala } \\
\text { (Roth) Alston }\end{array}$ & Leguminaceae & $\mathrm{P} 1$ & & & & & $\sqrt{ }$ & $\sqrt{ }$ & $\sqrt{ }$ & & & \\
\hline $\begin{array}{l}\text { Trumpet } \\
\text { vine }\end{array}$ & $\begin{array}{l}\text { Campsis } \\
\text { grandiflora } \\
\text { (Thunb.) K. } \\
\text { Schum. }\end{array}$ & Bignoniaceae & N1P2 & & & $\sqrt{ }$ & $\sqrt{ }$ & $\sqrt{ }$ & $\sqrt{ }$ & & & & \\
\hline $\begin{array}{l}\text { Cannabis } \\
\text { (Ghanja) }\end{array}$ & $\begin{array}{l}\text { Cannabis } \\
\text { sativa } \mathrm{L} .\end{array}$ & Cannabaceae & $\mathrm{N} 2 \mathrm{P} 2$ & & & & $\sqrt{ }$ & $\sqrt{ }$ & & & & & \\
\hline
\end{tabular}


[Rijal et. al., Vol.6 (Iss.11): November 2018]

(Received: October 23, 2018 - Accepted: November 25, 2018)

DOI: 10.5281/zenodo.1928975

\begin{tabular}{|c|c|c|c|c|c|c|c|c|c|c|c|c|c|c|}
\hline Clotoria & $\begin{array}{l}\text { Clitoria } \\
\text { ternatea } \mathrm{L} .\end{array}$ & Leguminaceae & $\mathrm{N} 2 \mathrm{P} 2$ & & & & & & $\sqrt{ }$ & & & & & \\
\hline Coffee & $\begin{array}{l}\text { Coffea } \\
\text { arabica } \mathrm{L} .\end{array}$ & Rubiaceae & $\mathrm{N} 2 \mathrm{P} 2$ & & $\sqrt{ }$ & $\sqrt{ }$ & & & & & & & & \\
\hline Mayan leaf & $\begin{array}{l}\text { Coleus } \\
\text { blumei Benth. }\end{array}$ & Lamiaceae & $\mathrm{N} 2$ & & & & & $\sqrt{ }$ & $\sqrt{ }$ & $\sqrt{ }$ & & & & \\
\hline Duranta & $\begin{array}{l}\text { Duranta } \\
\text { erecta L. }\end{array}$ & Verbenaceae. & $\mathrm{P} 2$ & & $\sqrt{ }$ & $\sqrt{ }$ & & & & & & & & \\
\hline Rudraksha & $\begin{array}{l}\text { Elaeocarpus } \\
\text { sphericus } \\
\text { (Gsertn) K. } \\
\text { Schum. }\end{array}$ & $\begin{array}{l}\text { Elaeocarpacea } \\
\text { e }\end{array}$ & N1P2 & & & & $\sqrt{ }$ & & & & & & & \\
\hline Eucalyptus & $\begin{array}{l}\text { Eucalyptus } \\
\text { citriodora } \\
\text { Hook }\end{array}$ & Myrtaceae & $\mathrm{N} 2 \mathrm{P} 2$ & & & & & $\sqrt{ }$ & $\sqrt{ }$ & & & & & \\
\hline Euphorbia & $\begin{array}{l}\text { Euphorbia } \\
\text { hirta L. }\end{array}$ & $\begin{array}{l}\text { Euphorbiacea } \\
\mathrm{e}\end{array}$ & $\mathrm{P} 2$ & & & & $\sqrt{ }$ & $\sqrt{ }$ & $\sqrt{ }$ & & & & & \\
\hline Milkweed & $\begin{array}{l}\text { Launaea } \\
\text { asplenifolia } \\
\text { (Willd.) Hook } \\
\text { f. }\end{array}$ & Compositae & P1 & & & & $\sqrt{ }$ & $\sqrt{ }$ & $\sqrt{ }$ & & & & & \\
\hline Ficus & $\begin{array}{l}\text { Ficus } \\
\text { benghalensis } \\
\text { L. }\end{array}$ & Moraceae & N2 & & $\sqrt{ }$ & & & & & & & & & \\
\hline $\begin{array}{l}\text { Ficus } \\
\text { (Kabro) }\end{array}$ & $\begin{array}{l}\text { Ficus lacor } \\
\text { Buch.-Ham. }\end{array}$ & Moraceae & $\mathrm{N} 2$ & & $\sqrt{ }$ & & & & & & & & & \\
\hline $\begin{array}{l}\text { Cluster fig } \\
\text { tree (Dumri } \\
\text { ) }\end{array}$ & $\begin{array}{l}\text { Ficus } \\
\text { racemosa L. }\end{array}$ & Moraceae & $\mathrm{N} 2$ & & $\sqrt{ }$ & & & & & & & & & \\
\hline Pipal & $\begin{array}{l}\text { Ficus } \\
\text { religiosa L. }\end{array}$ & Moraceae & N1 & & $\sqrt{ }$ & & & & & & & & & \\
\hline Cotton & $\begin{array}{l}\text { Gossypium } \\
\text { hirsutum L. }\end{array}$ & Malbaceae & N1P2 & & & & & & & & $\sqrt{ }$ & & & \\
\hline Jacanranda & $\begin{array}{l}\text { Jacanranda } \\
\text { mimosifolia } \\
\text { D. Don. }\end{array}$ & Bignoniaceae & N1P1 & & & & $\sqrt{ }$ & $\sqrt{ }$ & & & & & & \\
\hline Jatropha & $\begin{array}{l}\text { Jatropha } \\
\text { curcas L. }\end{array}$ & $\begin{array}{l}\text { Euphorbiacea } \\
\mathrm{e}\end{array}$ & $\mathrm{N} 2$ & & & & $\sqrt{ }$ & $\sqrt{ }$ & & & & & & \\
\hline Pumello & $\begin{array}{l}\text { Maesa } \\
\text { macrophylla } \\
\text { Wall. }\end{array}$ & Myrsinaceae & N1P1 & $\sqrt{ }$ & $\sqrt{ }$ & $\sqrt{ }$ & & & & & & & & \\
\hline Momordica & $\begin{array}{l}\text { Momordica } \\
\text { balsamina L. }\end{array}$ & Cucurbitaceae & $\mathrm{P} 2$ & $\sqrt{ }$ & $\sqrt{ }$ & & & & & & & & & \\
\hline Moringa & $\begin{array}{l}\text { Moringa } \\
\text { oleifera Lam. }\end{array}$ & Moringaceae & N1P1 & $\sqrt{ }$ & $\sqrt{ }$ & & & & & & & & & \\
\hline $\begin{array}{l}\text { Poison ivy } \\
\text { (Bhalayo) }\end{array}$ & $\begin{array}{l}\text { Rhus } \\
\text { wallichii } \\
\text { Hook.f. }\end{array}$ & $\begin{array}{l}\text { Anacardiacea } \\
\mathrm{e}\end{array}$ & $\mathrm{N} 2 \mathrm{P} 2$ & & & & & $\sqrt{ }$ & $\sqrt{ }$ & $\sqrt{ }$ & $\sqrt{ }$ & & & $\sqrt{ }$ \\
\hline $\begin{array}{l}\text { Richinus } \\
\text { (Aadir) }\end{array}$ & $\begin{array}{l}\text { Richinus } \\
\text { communis L. }\end{array}$ & $\begin{array}{l}\text { Euphorbiacea } \\
\mathrm{e}\end{array}$ & $\mathrm{N} 2 \mathrm{P} 2$ & & & & $\sqrt{ }$ & $\sqrt{ }$ & $\sqrt{ }$ & $\sqrt{ }$ & & & & \\
\hline
\end{tabular}




\begin{tabular}{|c|c|c|c|c|c|c|c|c|c|c|c|c|c|c|c|}
\hline Sapium & $\begin{array}{l}\text { Sapium } \\
\text { insigne } \\
\text { (Royale) } \\
\text { Benth.ex. } \\
\text { Hook.f. }\end{array}$ & $\begin{array}{l}\text { Euphorbiacea } \\
\text { e }\end{array}$ & N1 & & & & & & $\sqrt{ }$ & $\sqrt{ }$ & & & & & \\
\hline Schima & $\begin{array}{l}\text { Schima } \\
\text { wallichii } \\
\text { (DC) Korth. }\end{array}$ & Theaceae & $\mathrm{N} 2 \mathrm{P} 2$ & & & & & $\sqrt{ }$ & $\sqrt{ }$ & & & & & & \\
\hline $\begin{array}{l}\text { Broomweed } \\
\text { (Chini Jhar) }\end{array}$ & $\begin{array}{l}\text { Scoparia } \\
\text { dulcis L. }\end{array}$ & $\begin{array}{l}\text { Plantaginacea } \\
\mathrm{e}\end{array}$ & $\mathrm{P} 2$ & & & & $\sqrt{ }$ & $\sqrt{ }$ & $\sqrt{ }$ & & & & & & \\
\hline Sechium & $\begin{array}{l}\text { Sechium } \\
\text { edule (Jacq.) } \\
\text { Sw. }\end{array}$ & Cucurbitaceae & $\mathrm{N} 2 \mathrm{P} 2$ & & & & & & & & $\sqrt{ }$ & $\sqrt{ }$ & 1 & $\sqrt{ }$ & $\sqrt{ }$ \\
\hline Tik & $\begin{array}{l}\text { Tectona } \\
\text { grandis L.f. }\end{array}$ & Lamiaceae & $\mathrm{N} 2 \mathrm{P} 2$ & & & $\sqrt{ }$ & & & & & & & & & \\
\hline $\begin{array}{l}\text { Black } \\
\text { mayobalan } \\
\text { (Barro) }\end{array}$ & $\begin{array}{l}\text { Terminalia } \\
\text { chebula } \text { Retz. }\end{array}$ & Combretaceae & N1P1 & & & & $\sqrt{ }$ & $\sqrt{ }$ & & & & & & & \\
\hline Vitex & $\begin{array}{l}\text { Vitex } \\
\text { negunod L. }\end{array}$ & Lamiaceae & N1P2 & & & & $\sqrt{ }$ & $\sqrt{ }$ & & & & & & & \\
\hline $\begin{array}{l}\text { Woodfordia } \\
\text { (Dhangero) }\end{array}$ & $\begin{array}{l}\text { Woodfordia } \\
\text { fruticosa } \\
\text { (L.)Kurz. }\end{array}$ & Lythraceae & N1P1 & & & $\sqrt{ }$ & $\sqrt{ }$ & & & & & & & & \\
\hline $\begin{array}{l}\text { Adina } \\
(\text { Karam) }\end{array}$ & $\begin{array}{l}\text { Adina } \\
\text { cardifolia } \\
\text { (Willd. ex } \\
\text { Roxb.) Benth. } \\
\text { and Hook.f. } \\
\text { ex Brandis }\end{array}$ & Rubiaceae & $\mathrm{N} 2 \mathrm{P} 2$ & & & $\sqrt{ }$ & $\sqrt{ }$ & & & & & & & & \\
\hline $\begin{array}{l}\text { Litsea } \\
\text { (Kutmiro) }\end{array}$ & $\begin{array}{l}\text { Litsea } \\
\text { monopetala } \\
\text { (Roxb.) Pers. }\end{array}$ & Lauraceae & $\mathrm{N} 2 \mathrm{P} 2$ & $\sqrt{ }$ & $\sqrt{ }$ & $\sqrt{ }$ & $\sqrt{ }$ & $\sqrt{ }$ & $\sqrt{ }$ & & & & & & \\
\hline $\begin{array}{l}\text { Wild } \\
\text { eggplant }\end{array}$ & $\begin{array}{l}\text { Solanum } \\
\text { torvum } \\
\text { Swartz. }\end{array}$ & Solanaceae & $\mathrm{P} 2$ & $\sqrt{ }$ & $\sqrt{ }$ & $\sqrt{ }$ & $\sqrt{ }$ & $\sqrt{ }$ & $\sqrt{ }$ & $\sqrt{ }$ & $\sqrt{ }$ & $\sqrt{ }$ & 1 & $\sqrt{ }$ & $\sqrt{ }$ \\
\hline Love apple & $\begin{array}{l}\text { Solanum } \\
\text { aculeatissimu } \\
\text { m Jacq. }\end{array}$ & Solanaceae & $\mathrm{P} 2$ & & & & & & & $\sqrt{ }$ & $\sqrt{ }$ & $\sqrt{ }$ & 1 & & \\
\hline $\begin{array}{l}\text { Chaff- } \\
\text { flower } \\
\text { (Apamarga) }\end{array}$ & $\begin{array}{l}\text { Achyranthes } \\
\text { aspera } \mathrm{L} .\end{array}$ & $\begin{array}{l}\text { Amaranthacea } \\
\text { e }\end{array}$ & $\mathrm{N} 2$ & & & & & & & & & $\sqrt{ }$ & 1 & $\sqrt{ }$ & $\sqrt{ }$ \\
\hline $\begin{array}{l}\text { Dwarf } \\
\text { copperleaf } \\
\text { (Bhirangi } \\
\text { jhar) }\end{array}$ & $\begin{array}{l}\text { Alternanther } \\
\text { a sessilis (L.) } \\
\text { R.Br.ex DC. }\end{array}$ & $\begin{array}{l}\text { Amaranthacea } \\
\mathrm{e}\end{array}$ & $\mathrm{N} 2 \mathrm{P} 2$ & & & & & & & & & $\sqrt{ }$ & 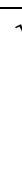 & & \\
\hline $\begin{array}{l}\text { Cacia tora } \\
\text { (small) }\end{array}$ & $\begin{array}{l}\text { Cassia tora } \\
\text { L. }\end{array}$ & Leguminaceae & $\mathrm{P} 2$ & & & & & & & $\sqrt{ }$ & $\sqrt{ }$ & & & & \\
\hline Hydrocotyle & $\begin{array}{l}\text { Hydrocotyle } \\
\text { nepalensis } \\
\text { Hooker. }\end{array}$ & Araliaceae & $\mathrm{N} 2 \mathrm{P} 2$ & & & & & & & $\sqrt{ }$ & $\sqrt{ }$ & & & & \\
\hline
\end{tabular}




\begin{tabular}{|c|c|c|c|c|c|c|c|c|c|c|c|c|c|c|c|}
\hline Digitaria & $\begin{array}{l}\text { Digitaria } \\
\text { ciliaris } \\
\text { (Retz.) } \\
\text { Koeler. }\end{array}$ & Poaceae & $\mathrm{N} 2$ & & & & & & $\sqrt{ }$ & & & & & & \\
\hline Cyperus & $\begin{array}{l}\text { Cyperus } \\
\text { rotundus L. }\end{array}$ & Cyperaceae & $\mathrm{P} 2$ & & & & & & & & $\sqrt{ }$ & $\sqrt{ }$ & $\sqrt{ }$ & & \\
\hline $\begin{array}{l}\text { Prickly } \\
\text { poppy }\end{array}$ & $\begin{array}{l}\text { Argemone } \\
\text { mexicana } \mathrm{L} .\end{array}$ & Papavaraceae & N1P1 & & & & & & & & $\sqrt{ }$ & $\sqrt{ }$ & $\sqrt{ }$ & & \\
\hline Lotus & $\begin{array}{l}\text { Nelumbo } \\
\text { nucifera } \\
\text { Gaertn. }\end{array}$ & $\begin{array}{l}\text { Nelumbonace } \\
\text { ae }\end{array}$ & $\mathrm{P} 1$ & & & & & & & $\sqrt{ }$ & $\sqrt{ }$ & $\sqrt{ }$ & & & \\
\hline $\begin{array}{l}\text { Thatch } \\
\text { grass }\end{array}$ & $\begin{array}{l}\text { Saccharum } \\
\text { spontaneum } \\
\text { L. }\end{array}$ & Poaceae. & $\mathrm{P} 2$ & & & & & & & & $\sqrt{ }$ & $\sqrt{ }$ & $\sqrt{ }$ & & \\
\hline Whip grass & $\begin{array}{l}\text { Hemarthria } \\
\text { compressa } \\
\text { (L.f.) R.Br. }\end{array}$ & Poaceae & $\mathrm{P} 1$ & $\sqrt{ }$ & $\sqrt{ }$ & & $\sqrt{ }$ & $\sqrt{ }$ & $\sqrt{ }$ & $\sqrt{ }$ & $\sqrt{ }$ & $\sqrt{ }$ & $\sqrt{ }$ & $\sqrt{ }$ & $\sqrt{ }$ \\
\hline Imperata & $\begin{array}{l}\text { Imperata } \\
\text { cylindrica } \\
\text { (L.) P.Beauv. }\end{array}$ & Poaceae & $\mathrm{P} 2$ & & & & & & & & & $\sqrt{ }$ & $\sqrt{ }$ & $\sqrt{ }$ & \\
\hline Saccharum & $\begin{array}{l}\text { Saccharum } \\
\text { bengalense } \\
\text { Retz. }\end{array}$ & Poaceae & $\mathrm{P} 2$ & & $\sqrt{ }$ & & & & & & & & & & \\
\hline Calicarpa & $\begin{array}{l}\text { Calicarpa } \\
\text { macrophylla } \\
\text { Vahl. }\end{array}$ & Verbenaceae & $\mathrm{N} 2 \mathrm{P} 1$ & & & & & & & & & & & & \\
\hline Dhursil & $\begin{array}{l}\text { Colebrookea } \\
\text { oppositifolia } \\
\text { Smith }\end{array}$ & Labiatceae & $\mathrm{P} 2$ & $\sqrt{ }$ & $\sqrt{ }$ & & & & & & & & & & $\sqrt{ }$ \\
\hline Kadipatta & $\begin{array}{l}\text { Murraya } \\
\text { koenigii (L.) } \\
\text { Spreng. }\end{array}$ & Rutaceae & N1P1 & & & & & & $\sqrt{ }$ & & & & & & \\
\hline $\begin{array}{l}\text { Lantana } \\
\text { (Yellow) }\end{array}$ & $\begin{array}{l}\text { Lantana } \\
\text { camara } \mathrm{L} .\end{array}$ & Verbenaceae & $\mathrm{N} 2 \mathrm{P} 2$ & $\sqrt{ }$ & $\sqrt{ }$ & & $\sqrt{ }$ & $\sqrt{ }$ & $\sqrt{ }$ & $\sqrt{ }$ & & & & & \\
\hline Coccinea & $\begin{array}{l}\text { Coccinea } \\
\text { grandis (L.) } \\
\text { Voigt }\end{array}$ & Cucurbitaceae & N1P2 & & & & & & $\sqrt{ }$ & $\sqrt{ }$ & & & & & \\
\hline Wild radish & $\begin{array}{l}\text { Blumea } \\
\text { lacera (Burn. } \\
\text { f.) DC }\end{array}$ & Asteraceae & $\mathrm{N} 2 \mathrm{P} 2$ & $\sqrt{ }$ & $\sqrt{ }$ & & & & & & & & & & $\sqrt{ }$ \\
\hline Calotropis & $\begin{array}{l}\text { Calotropis } \\
\text { gigantea (L.) } \\
\text { W.T.Alton }\end{array}$ & Apocynaceae & $\mathrm{P}$ & $\sqrt{ }$ & $\sqrt{ }$ & & & & & & & & & & \\
\hline Dhaincha & $\begin{array}{l}\text { Sesbania } \\
\text { grandiflora } \\
\text { (L.) Poiret. }\end{array}$ & Fabaceae & $\mathrm{P} 1$ & & & & & & & & & & $\sqrt{ }$ & $\sqrt{ }$ & \\
\hline Wild bean & $\begin{array}{l}\text { Ceropegia } \\
\text { pubescens } \\
\text { Wall. }\end{array}$ & $\begin{array}{l}\text { Asclepiadacea } \\
\mathrm{e}\end{array}$ & $\mathrm{N} 2 \mathrm{P} 2$ & $\sqrt{ }$ & $\sqrt{ }$ & & & & & & & & & & \\
\hline
\end{tabular}




\begin{tabular}{|c|c|c|c|c|c|c|c|c|c|c|c|c|c|c|c|}
\hline Piper & $\begin{array}{l}\text { Piper longum } \\
\text { L. }\end{array}$ & Piperaceae & $\mathrm{P} 2$ & & & & & & & & $\sqrt{ }$ & $\sqrt{ }$ & & & \\
\hline $\begin{array}{l}\text { Wild } \\
\text { temeric }\end{array}$ & $\begin{array}{l}\text { Curcuma } \\
\text { aromatica } \\
\text { Salisb. } \\
\end{array}$ & Zingiberaceae & $\mathrm{N} 2 \mathrm{P} 2$ & & & & & & & & & & & & \\
\hline Mikania & $\begin{array}{l}\text { Mikania } \\
\text { micarantha } \\
\text { Kunth }\end{array}$ & Asteraceae & $\mathrm{N} 2 \mathrm{P} 2$ & & $\sqrt{ }$ & & & & & & & & & & \\
\hline Paspalum & $\begin{array}{l}\text { Paspalum } \\
\text { distichum } \mathrm{L} .\end{array}$ & Poaceae & $\mathrm{P} 2$ & & & & & & & & $\sqrt{ }$ & $\sqrt{ }$ & & & \\
\hline $\begin{array}{l}\text { Cynoglossu } \\
\mathrm{m}\end{array}$ & $\begin{array}{l}\text { Cynoglossum } \\
\text { glochidiatum } \\
\text { Wall.ex } \\
\text { Benth }\end{array}$ & Boraginaceae & $\mathrm{P} 2$ & & & & & & $\sqrt{ }$ & $\sqrt{ }$ & & & & & \\
\hline Croton & $\begin{array}{l}\text { Croton } \\
\text { sparciflora } \\
\text { Morong } \\
\end{array}$ & $\begin{array}{l}\text { Euphorbiacea } \\
\text { e }\end{array}$ & $\begin{array}{l}\mathrm{N} 2 \\
\mathrm{P} 2\end{array}$ & $\sqrt{ }$ & $\sqrt{ }$ & & & & & & & & & & \\
\hline $\begin{array}{l}\text { Achyranthe } \\
\mathrm{s}\end{array}$ & $\begin{array}{l}\text { Achyranthes } \\
\text { aspera L. }\end{array}$ & $\begin{array}{l}\text { Amaranthacea } \\
\text { e. }\end{array}$ & $\mathrm{N} 2 \mathrm{P} 2$ & & & & & & $\sqrt{ }$ & $\sqrt{ }$ & & & & & \\
\hline Datura & $\begin{array}{l}\text { Datura } \\
\text { stramonium } \\
\text { L. } \\
\end{array}$ & Solanaceae & $\mathrm{P} 2$ & & & & & $\sqrt{ }$ & $\sqrt{ }$ & & & & & & \\
\hline $\begin{array}{l}\text { Clerodendru } \\
\mathrm{m}\end{array}$ & $\begin{array}{l}\text { Clerodendru } \\
m \\
\text { infortunatum } \\
\text { L. }\end{array}$ & Lamiaceae & N1 & & & & $\sqrt{ }$ & $\sqrt{ }$ & & & & & & & \\
\hline $\begin{array}{l}\text { pignut Wild } \\
\text { ( sesame }\end{array}$ & $\begin{array}{l}\text { Hyptis } \\
\text { suaveolens } \\
\text { (L.) Poit. } \\
\end{array}$ & Lamiaceae & $\mathrm{N} 2$ & & & & & & & & $\sqrt{ }$ & $\sqrt{ }$ & & & \\
\hline Vicia & $\begin{array}{l}\text { Vicia } \\
\text { angustifolia } \\
\text { (L.) ex Reich. }\end{array}$ & Leguminaceae & $\mathrm{P} 2$ & $\sqrt{ }$ & $\sqrt{ }$ & & & & & & & & & & \\
\hline Urtica & $\begin{array}{l}\text { Urtica dioica } \\
\text { L. }\end{array}$ & Urticaceae & $\mathrm{N} 2 \mathrm{P} 2$ & & $\sqrt{ }$ & $\sqrt{ }$ & & & & & & & & & \\
\hline Oxalis & $\begin{array}{l}\text { Oxalis } \\
\text { corniculata } \\
\text { L. }\end{array}$ & Oxalidaceae & $\mathrm{N} 2$ & & & & & $\sqrt{ }$ & $\sqrt{ }$ & $\sqrt{ }$ & $\sqrt{ }$ & $\sqrt{ }$ & $\sqrt{ }$ & $\sqrt{ }$ & \\
\hline Mallotus & $\begin{array}{l}\text { Mallotus } \\
\text { phillipensis } \\
\text { (Lam.) Muell. } \\
\text { Arg. }\end{array}$ & $\begin{array}{l}\text { Euphorbiacea } \\
\mathrm{e}\end{array}$ & $\mathrm{N} 2 \mathrm{P} 2$ & & $\sqrt{ }$ & $\sqrt{ }$ & & & & & & & & & \\
\hline Grewia & $\begin{array}{l}\text { Grewia } \\
\text { helicterifolia } \\
\text { Wall.ex G. } \\
\text { Don. }\end{array}$ & Tiliaceae & $\mathrm{P} 2$ & & & & $\sqrt{1}$ & $\sqrt{ }$ & & & & & & & \\
\hline Calotropis & $\begin{array}{l}\text { Calotropis } \\
\text { gigantea (L.) } \\
\text { W.T.Aiton }\end{array}$ & $\begin{array}{l}\text { Asclepiadacea } \\
\text { e }\end{array}$ & $\mathrm{P} 1$ & $\sqrt{ }$ & $\sqrt{ }$ & & & & & & & & & & \\
\hline Lime grass & $\begin{array}{l}\text { Cympopogon } \\
\text { flexuosus }\end{array}$ & Poaceae & $\mathrm{N} 2 \mathrm{P} 2$ & $\sqrt{ }$ & $\sqrt{ }$ & & & & & & & & & & \\
\hline
\end{tabular}




\begin{tabular}{|c|c|c|c|c|c|c|c|c|c|c|c|c|c|c|}
\hline & $\begin{array}{lr}\text { Nees } & \text { ex } \\
\text { Steud.) } & \text { W. } \\
\text { Atson } & \\
\end{array}$ & & & & & & & & & & & & & \\
\hline $\begin{array}{l}\text { Common } \\
\text { mallow }\end{array}$ & $\begin{array}{l}\text { Thespesia } \\
\text { lampas (Cav.) } \\
\text { Dalz. \& A. } \\
\text { Gibson }\end{array}$ & Malvaceae & $\mathrm{P} 1 \mathrm{~N} 2$ & & $\sqrt{ }$ & $\sqrt{ }$ & & & & & & & & \\
\hline $\begin{array}{l}\text { Chaklatewe } \\
\text { ed } \\
\text { (Bankuro) }\end{array}$ & $\begin{array}{l}\text { Melochia } \\
\text { corchorifolia } \\
\text { L. }\end{array}$ & Malvaceae & $\mathrm{P} 2$ & & & & & & & & & & & \\
\hline Potentilla & $\begin{array}{l}\text { Potentilla } \\
\text { supina } \mathrm{L} .\end{array}$ & Rosaceae & $\mathrm{N} 2$ & & & & & $\sqrt{ }$ & $\sqrt{ }$ & & & & & \\
\hline Eclipta & $\begin{array}{l}\text { Eclipta } \\
\text { prostrata (L.) } \\
\text { L. }\end{array}$ & Asteraceae & $\mathrm{P} 2 \mathrm{~N} 2$ & $\sqrt{ }$ & $\sqrt{ }$ & & & & & & & & & \\
\hline Ixeris & $\begin{array}{l}\text { Ixeris } \\
\text { polycephala } \\
\text { Cass. }\end{array}$ & Asteraceae & $\mathrm{P} 2$ & & & & & & $\sqrt{ }$ & $\sqrt{ }$ & & & & \\
\hline Xanthium & $\begin{array}{l}\text { Xanthium } \\
\text { strumarium } \\
\text { L. }\end{array}$ & Asteraceae & $\mathrm{P} 2$ & & & & & & $\sqrt{ }$ & $\sqrt{ }$ & & & & \\
\hline $\begin{array}{l}\text { Garuga } \\
\text { (Dabdabe) }\end{array}$ & $\begin{array}{l}\text { Garuga } \\
\text { pinnata } \\
\text { Roxb. }\end{array}$ & Burseraceae & $\mathrm{N} 2 \mathrm{P} 2$ & & & & & $\sqrt{ }$ & $\sqrt{ }$ & & & & & \\
\hline Cyperus & Cyperus spp. & Cyperaceae & $\mathrm{P} 2$ & & & & $\sqrt{ }$ & $\sqrt{ }$ & & & & & & \\
\hline Eleocharis & $\begin{array}{l}\text { Eleocharis } \\
\text { pellucida } \mathrm{J} . \\
\text { Presl. \& C. } \\
\text { Presl. }\end{array}$ & Cyperaceae & $\mathrm{N} 2$ & & & & & & $\sqrt{ }$ & $\sqrt{ }$ & & & & \\
\hline Dillenia & $\begin{array}{l}\text { Dillenia } \\
\text { pentagyna } \\
\text { Roxb. }\end{array}$ & Dilleniaceae & N1P2 & & & $\sqrt{ }$ & $\sqrt{ }$ & & & & & & & \\
\hline Desmodium & $\begin{array}{l}\text { Ocimum } \\
\text { basilicum L. }\end{array}$ & Labiateae & $\mathrm{N} 2 \mathrm{P} 2$ & $\sqrt{ }$ & $\sqrt{ }$ & & & & & & & & & \\
\hline Phaseolus & $\begin{array}{l}\text { Desmodium } \\
\text { oojeinese } \\
\text { (Roxb.) H. } \\
\text { Ohashi }\end{array}$ & Leguminosae & N1P2 & & & & $\sqrt{ }$ & $\sqrt{ }$ & & & & & & \\
\hline Fragaria & $\begin{array}{l}\text { Flemingia } \\
\text { macrophylla } \\
\text { (Willd.) } \\
\text { Merr. }\end{array}$ & Leguminosae & $\mathrm{N} 2 \mathrm{P} 2$ & & & & & & & & $\sqrt{ }$ & $\sqrt{ }$ & & \\
\hline Xeromphis & $\begin{array}{l}\text { Phaseolus } \\
\text { mungo L. }\end{array}$ & Leguminosae & $\mathrm{N} 2 \mathrm{P} 2$ & & & & & & $\sqrt{ }$ & $\sqrt{ }$ & & & & \\
\hline Schleichera & $\begin{array}{l}\text { Fragaria } \\
\text { nubicola } \\
\text { Lindi.ex. } \\
\text { Lacaita }\end{array}$ & Rosaceae & N1 & & & & & $\sqrt{ }$ & $\sqrt{ }$ & & & & & \\
\hline Typha & $\begin{array}{l}\text { Xeromphis } \\
\text { uliginosa }\end{array}$ & Rubiaceae & $\mathrm{N} 2 \mathrm{P} 2$ & & & & & & $\sqrt{ }$ & $\sqrt{ }$ & & & & \\
\hline
\end{tabular}




\begin{tabular}{|c|c|c|c|c|c|c|c|c|c|c|c|c|c|c|}
\hline & $\begin{array}{l}\text { (Retz.) } \\
\text { Maheshw. }\end{array}$ & & & & & & & & & & & & & \\
\hline Phyla & $\begin{array}{l}\text { Schleichera } \\
\text { oleosa } \\
\text { (Lour.) Oken }\end{array}$ & Sapindaceae & $\mathrm{N} 2 \mathrm{P} 1$ & $\sqrt{ }$ & $\sqrt{ }$ & & & & & & & & & \\
\hline Wild okra & $\begin{array}{l}\text { Typha } \\
\text { angustifolia } \\
\text { L. }\end{array}$ & Typhaceae & $\mathrm{P} 1$ & & & & & & $\sqrt{ }$ & $\sqrt{ }$ & & & & \\
\hline $\begin{array}{l}\text { Sawtooth } \\
\text { fogfruit } \\
\text { (Paadke } \\
\text { Siris) } \\
\end{array}$ & $\begin{array}{l}\text { Phyla } \\
\text { nodiflora (L.) } \\
\text { Greene }\end{array}$ & Verbenaceae & N1 & & & $\sqrt{ }$ & $\sqrt{ }$ & $\sqrt{ }$ & $\sqrt{ }$ & $\sqrt{ }$ & $\sqrt{ }$ & $\sqrt{ }$ & $\sqrt{ }$ & $\sqrt{ }$ \\
\hline Aegle & $\begin{array}{l}\text { Flemingia } \\
\text { strobilifera } \\
\text { (L.) W.T. } \\
\text { Aiton }\end{array}$ & Fabaceae & $\mathrm{N} 2 \mathrm{P} 1$ & $\sqrt{ }$ & $\sqrt{ }$ & & & & & & & & & \\
\hline $\begin{array}{l}\text { Native } \\
\text { rosella } \\
\text { (Mulapate) }\end{array}$ & $\begin{array}{l}\text { Abelmoschus } \\
\text { ficulneus (L.) } \\
\text { Wight \& Arn. }\end{array}$ & Malvaceae & $\mathrm{N} 2 \mathrm{P} 2$ & & $\sqrt{ }$ & & & $\sqrt{ }$ & $\sqrt{ }$ & & & & & \\
\hline Pink sisis & $\begin{array}{l}\text { Albizia } \\
\text { julibrissin } \\
\text { (Var.) }\end{array}$ & Fabaceae & N1P1 & & & & & & & & & $\sqrt{ }$ & $\sqrt{ }$ & \\
\hline $\begin{array}{l}\text { Wood apple } \\
\text { (Mirchaiya) }\end{array}$ & $\begin{array}{l}\text { Aegle } \\
\text { marmelos } \\
\text { (L.) Correa } \\
\end{array}$ & Rutaceae & N1P2 & & & & $\sqrt{ }$ & & & & & & & \\
\hline $\begin{array}{l}\text { Batoko } \\
\text { plum }\end{array}$ & $\begin{array}{l}\text { Diplacus } \\
\text { inermis Wall } \\
\text { ex Roxb. }\end{array}$ & Lamiaceae & $\mathrm{N} 2 \mathrm{P} 2$ & & & & & & $\sqrt{ }$ & $\sqrt{ }$ & $\sqrt{ }$ & & & \\
\hline
\end{tabular}

* $\mathrm{H}=$ Herb, $\mathrm{T}=$ Tree, $\mathrm{S}=\mathrm{Shrub}$

$* * \mathrm{~N} 1=$ high nectar, $\mathrm{N} 2=$ low nectar, $\mathrm{P} 1=$ High pollen, $\mathrm{P} 2=$ low pollen

Rice, Oryza sativa L.; maize, Zea mays L.; buckwheat, Fagopyrum esculentum Moench.; rapeseed, Brassica campestris L.; sesame, Sesamum orientale L.; litchi, Litchi chinensis Sonner; .; guava, Pisum sativum L ; cucurbits (bottle gourd, Lagenaria siceraria (Molina) Standl; sponge gourd, Luffa cylindrica (L.) Roem.; bitter gourd, Momordica charantia L.; Leucas (gumpate), Leucas lanata Benth.; Butternut (chiuri), Bassia butyracea Roxb: Pogostemon (rudilo), Pogostemon glaber BenthSisoo, Dalbergia sissoo Roxb.; Throughwort (Banmara), Eupatorium sp. and silk tree (Padke), Albizia julibrissin Durazz; Murraya (kadipatta) Murraya koenigii (L.) Spreng. are the major bee forage species of the district. Fagopyrum esculentum Moench, Brassica campestral L, Lagerstroemia indica L, Bombax ceiba L, Albizia julibrissin Durazz., Eugenia fprmosa Wall., Shorea robusta, Guartn. Upatorium grandulosum, Pogostemon glaber Benth. Terminalia alata Heyne ex.Roth, Murraya koenigii (L.) Spreng Sesamum orientale L. Dalbergia sissoo Roxb. were the major bee flora both rich in nectar and pollen.

Table 3: Distribution of crops and wild bee forage plant in different months in Chitwan, during 2012-2013

\begin{tabular}{|l|l|l|l|l|l|l|l|l|l|l|l|l|}
\hline Particulars & Jan & Feb & Mar & Apr & May & Jun & July & Aug & Sept & Oct & Nov & Dec \\
\hline Cereal & 3 & 2 & 2 & 0 & 2 & 2 & 1 & 5 & 4 & 3 & 3 & 1 \\
\hline
\end{tabular}




\begin{tabular}{|l|l|l|l|l|l|l|l|l|l|l|l|l|}
\hline Pulses & 1 & 2 & 2 & 0 & 2 & 2 & 1 & 5 & 4 & 3 & 3 & 1 \\
\hline Oilseed & 2 & 4 & 2 & 2 & 1 & 1 & 3 & 2 & 3 & 2 & 1 & 2 \\
\hline Vegetable & 4 & 10 & 14 & 13 & 10 & 10 & 7 & 8 & 6 & 6 & 3 & 3 \\
\hline Fruits & 1 & 5 & 16 & 16 & 7 & 4 & 1 & 1 & 1 & 1 & 1 & 1 \\
\hline Ornamental & 6 & 7 & 7 & 9 & 13 & 15 & 14 & 14 & 12 & 12 & 8 & 8 \\
\hline Wild & 22 & 33 & 35 & 45 & 48 & 53 & 48 & 36 & 33 & 25 & 15 & 9 \\
\hline Total & 39 & 63 & 78 & 85 & 83 & 87 & 75 & 71 & 63 & 52 & 34 & 25 \\
\hline
\end{tabular}

Table 3 summaries the crops and wild bee forage with their flowering months, which indicated that majority of bee forage available in Feb-September. Though rainy season is drought period in Chitwan condition, many wild plant species found flowering in the season. Migration of bee colony in different places within Chitwan can lower the feeding to the bees in winter and rainy seasons.

Table 4: Bee forage plants with nectar and pollen sources found in Chitwan, Nepal during 2012-

\begin{tabular}{|l|l|l|l|l|l|l|l|l|l|}
\hline Particuclars & P1 & P2 & N1 & N2 & N1P1 & N2P1 & N1P2 & N2P2 & Total \\
\hline Cereals & 1 & 4 & 0 & 0 & 1 & 0 & 0 & 0 & 6 \\
\hline Wild plants & 11 & 32 & 10 & 16 & 23 & 6 & 10 & 47 & 155 \\
\hline Ornamentals & 1 & 3 & 2 & 2 & 5 & 0 & 3 & 10 & 26 \\
\hline Fruits & 0 & 2 & 0 & 1 & 11 & 2 & 1 & 4 & 21 \\
\hline Vegetables & 5 & 6 & 0 & 2 & 3 & 0 & 0 & 9 & 25 \\
\hline Pulses & 0 & 4 & 1 & 3 & 1 & 0 & 0 & 2 & 11 \\
\hline Oilseeds & 0 & 0 & 0 & 0 & 6 & 0 & 0 & 2 & 8 \\
\hline Total & 18 & 51 & 13 & 24 & 50 & 8 & 14 & 74 & 252 \\
\hline
\end{tabular}

Plants identified as bee forage are categorized as source of pollen (P1, P2) or nectar (N1, N2) and their combinations with their status in the study areas of Chitwan (Table 2). This includes 50 plant species rich in both nectar and pollen (N1P1), 14 species N1P2, 8 species N2P1, 74 species N2P2, 18 species rich in pollen only( $\mathrm{P} 10$, and 51 species as minor source of pollen (P2), 13 species rich in nectar (N1) and 24 species low nectar( N2). Pollen is necessary for brood development which were found to a greater amount in wild plants compared to crops. However, most of the wild plants which had low content of nectar and pollen.

Table 5: Bee forage crops and wild plants having different sources available in different months during 2012-2013

\begin{tabular}{|l|l|l|l|l|l|l|l|l|l|l|l|l|l|}
\hline Sources & Jan & Feb & Mar & Apl & May & Jun & Jul & Aug & Sept & Oct & Nov & Dec & $\begin{array}{l}\text { No. of } \\
\text { flora }\end{array}$ \\
\hline N1P1 & 7 & 15 & 26 & 27 & 12 & 12 & 8 & 7 & 6 & 8 & 6 & 5 & 50 \\
\hline N1 & 1 & 0 & 2 & 6 & 8 & 6 & 5 & 5 & 3 & 2 & 1 & 1 & 13 \\
\hline N2 & 4 & 5 & 6 & 2 & 4 & 6 & 3 & 5 & 5 & 8 & 7 & 4 & 24 \\
\hline P1 & 6 & 6 & 3 & 4 & 5 & 4 & 8 & 7 & 7 & 6 & 4 & 3 & 18 \\
\hline P2 & 9 & 14 & 14 & 13 & 16 & 15 & 14 & 15 & 15 & 9 & 9 & 5 & 51 \\
\hline N1P2 & 0 & 0 & 2 & 5 & 7 & 6 & 6 & 5 & 4 & 4 & 0 & 0 & 14 \\
\hline
\end{tabular}




\begin{tabular}{|l|l|l|l|l|l|l|l|l|l|l|l|l|l|}
\hline N2P1 & 2 & 2 & 1 & 2 & 2 & 2 & 1 & 1 & 0 & 0 & 1 & 1 & 8 \\
\hline N2P2 & 22 & 33 & 35 & 45 & 48 & 53 & 48 & 36 & 33 & 25 & 15 & 9 & 74 \\
\hline Total & 51 & 75 & 89 & 104 & 102 & 104 & 93 & 81 & 73 & 62 & 43 & 28 & 252 \\
\hline
\end{tabular}

Table 5 shows that bee forage were find well distributed throughout the year, however most of the species got flowering from Feb to September. Less number of plants were got flowering in November- January.

\section{Discussions}

Chitwan is rich in bee flora, i.e. 252 bee forage species were found during study. Most of these species were included in plant species lists in several other studies of Dangol and Shivakoti (2001), Thapa (2006) and Pokhrel, (2005) . Many crop and wild plants visited by bees in Chitwan include: litchi, buckwheat, rapeseed, Indian mustard, cowpea, radish, broccoli, sponge gourd, cucumber, brinjal, red gram, okra, mango, citrus, squash, bottlebrush, sesame and bottle gourd as reported by many workers (Thapa, 2002; Dhakal, 2003; Neupane, 2000; Devkota, 2000). The availability of major honey potential flora has led to great scope for beekeeping in the district. This is further supported by the availability of minor flora which mainly consists of wild flora which supports bee even in the dearth periods. This study was concurrence with the floral study of Kavre and Dolakha (Bista and Shivakoti, 2001) and Pokhrel (2005), respectively. Bista and Shivakoti (2001) at Kabre, Dolakha district indicated that the peak periods of honeybee foraging activity and abundant bee floral plants were recorded during mid-February and May (spring season); whereas from mid-November to February (winter season) is dearth period and the colony strength can be weak However, availability of honey potential bee flora is shown comparatively lacking in summer, which was also similar to the study of Mishra (1997/1998). Less honey potential floral sources are available to bees from June to September, so these months are very harsh for bees. Higher numbers of bee flora recorded in Megauli under semi- natural, shrub land and watershed areas found positive impact in biodiversity conservation as indicated by Emiru et al. (2006), in eastern zone of Tigray, Ethiopia. Jacobs et al. (2006) emphasized on bee flora species and other multipurpose species for rehabilitation of degraded and watershed areas, which were seen in Megauli. Akratanakul (1990) also lists guidelines for the exploration and evaluation of potential area for beekeeping with honeybee plants having relatively long blossoming periods, generally in terms of several weeks or months.

Knowledge about bee flora with their flowering time is very crucial for the farmers. Figure 2 shows that cultivated and wild bee forage plants bloomed in different months, however, the potential source of nectar and pollen were found in November to April, known as honey flow seasons in Chitwan condition. Honey flow is primarily due to the sufficiency of bee flora rapeseed, buckwheat and Bassia butyracea (Nov-Feb), Pogostemon sp. (Nov-Feb), Dalbergia sp. (AprilMay). Albizia sp. (July-Aug) Morraya sp (June), Eupatorium sp. (May-July), Bombax sp. (MarchApril), Termenalia sp( June-July), in different parts of Chitwan district. Several wild flora with minor honey sources were also found in rainy months (June-September) too, however, it was not sufficient for honeybee growth and development hence these period was taken as dearth period to bees in Chitwan condition. The cropland showed potentiality of increasing production and productivity by placing supplemental honeybee colonies on crop fields during flowering period as repoted by Garratt et al. (2013) in orchard fruit crops (Bommarco, Marini and Vaissi ere, (2012) 
in small fruits and vegetables (Andersson, Rundlof and Smith, 2012) and Partap C (1997) in fruit and seed production. However, this practice was not followed by the farmers resulting in low crop productivity there. Studies done by Partap and Partap (2002) and Thapa (2002) reveals that level of knowledge about biodiversity conservation, pollination and pollinators in Nepalese farmers are inadequate.

\section{Conclusion and Recommendation}

A total of 252 common bee flora species were identified in four study areas of Chitwan. The common bee forage species identified included: rice, maize, buckwheat, cowpea, black gram, rapeseed, mustard, and cucurbits, Lichi, citrus, Eupatorium, Pogostemon, Sissoo, Acacia, Termenalia, Albizia Morraya (kadipatta), Leucas. Most of the important bee flora was blooming from November to March, and this was peak period for honeybee foraging activity as well as peak time of honey harvest. There was shortage of bee forage in winter (Nov - January) and rainy period (June- Aug). There was variation in bee flora species diversity and abundance in different land use system, i.e. somewhat fewer floral species were recorded in organic site, i.e. Fulbari VDC, which was far from natural forests. In the study areas, there was good availability of bee forage in the summer season also. This was one of the positive impact of conservation of National Park, community forests, watershed and fallow lands. Though the sufficient bee flora was available in intensive agriculture practiced area, farmer kept away their hives in other places for foraging due to heavy use of pesticide in their crop fields. Hence, awareness raising program should be organized to educate them about importance of bee flora in pollinators and honey production. However, in the dry season only few bee flora plants were in bloom and this affected the colony performance. Therefore, to solve this problem beekeeping based agro-forestry practices on cultivated land, and selection of plant species that can tolerate drought and set flower for a long period should be introduced. Introduction of similar major honey producing plants in other countries or regions with similar vegetation patterns, agro-ecosystems, climate, and edaphic conditions also seemed necessary. Emphasis must be given honey potential plant which blooms longer period rather than covering large area. The large-scale planting of honeybee forages should be integrated with other agricultural activities, such as reforestation, roadside plantings, and animal pasture.

\section{Acknowledgements}

My sincere thanks goes to the Dean Prof. Dr. Keshav Raj Adhikari and Asst. Dean (Academic), Prof. Dr. Gopal Bahadur K.C. of IAAS, Tribhuvan University, Nepal for their valuable suggestions and support. I express my gratitude to Mr. Milan Subedi, Assistant Professor of IAAS, TU, Nepal for his regular support and motivation. I am thankful to all the farmers' groups and key informants of the research areas without their help the research will not be completed.

\section{References}

[1] Adhikari, S. and N.B. Ranabhat. 2003. Bee flora in mid hills of central Nepal. Botanica orientalisJ. Plant Sci. 8: 45-56.

[2] Akratanakul, P. 1990. Beekeeping in Asia. Food and Agriculture Organization of the United Nations, Agricultural Services. Bulletin 68/4. Rome, Italy. 
[3] Andersson, G.K.S., M. Rundlöf and H.G. Smith. 2012. Organic farming improves pollination success in strawberries. PLoS ONE 7: (2) e31599.

[4] Baptist, B.A. and R.W.K. Punchihewa .1980. A preliminary analysis of the principal factors which will affect apiary honey production in Sri Lanka. Proceedings of the 2nd Conference of Apiculture in Tropical Climates, New Delhi: 75-81

[5] Bartomeus, I., S.G. Potts, I. Steffan-Dewenter, B.E. Vaissière, M. Woyciechowski, K.M. Krewenka, T. Tscheulin, S.P.M. Roberts, H. Szentgyörgyi, C. Westphal and R. Bommarco. 2014. Contribution of insect pollinators to crop yield and quality varies with agricultural intensification. PeerJ 2: e328.

[6] Biesmeijer, J.C., S.P. Roberts, R. Reemer, M, Ohlemüller, M. Edwards , T. Peeters , A.P. Schaffers, S.G. Potts , R . Kleukers, C.D. Thomas, J. Settele and W.E. Kunin . 2006. Parallel declines in pollinators and insect-pollinated plants in Britain and the Netherlands. Science 313:351-354.

[7] Bedru, B., B. Muys and E. Mathiji. 2006. Economic valuation methods of forest rehabilitation in exclosures. J. Drylands 1(2):165-170.

[8] Bista S. and P. G. Shivakoti. 2001. Honeybee flora at Kabre Dolakha District, Nepal. Journal of Nepal Agric. Res. (4 \& 5): 16-25.

[9] Bommarco, R., L. Marini and B.E. Vaissiere. 2012. Insect pollination enhances seed yield, quality, and market value in oilseed rape. Oecologia 169: 1025-1035.

[10] Breeze, T.D., A.P. Bailey, K.G. Balcombe and S.G. Potts. 2010. Pollination services in the UK: How important are honeybees? Agri.Eco.Environ. 142 (3-4):137-143.

[11] Cameron, S. A., J. D. Lozier, J. P. Strange, J. B. Koch, N. Cordes, L. F. Solter, T. L. Griswold, and G. E. Robinson. 2011. Patterns of widespread decline in North American bumble bees. Proc. Natl. Acad. Sci. USA 108: 662-667.

[12] Costanza, R.; R. D’Arge, R. De Groot, S. Farber, M. Grasso, B. Hannon, K. Limburg, S. Naeem, R.V. O’Neil, J. Paruelo, P. Raskin, and M.van der Belt, 1997. The value of the world's ecosystem services. Nature 387: 253-260.

[13] Dangol, D. R. 2000. Studies on ethnobotany of weeds in Chitwan. pp. 123-135. Shrestha, S. M. and N. R.

[14] Devkota (eds.), IAAS Research Reports (1995-2000). Institute of Agriculture and Animal Science, Rampur.

[15] Dangol, D. R. and G.P. Shivakoti. 2001. Plant diversity of western Chitwan: A floristic approach. J. Nat.Hist. Mus. 20: 129-147

[16] Dhakal, G. 2003. Efficciency of Apis mellifera L. and Apis cerana F. for pollinating mustard and buckwheat. M.Sc. Thesis, Institute of Agriculture and Animal Sciences, Rampur, Chitwan, Nepal

[17] Devkota, F.P. 2000. Comparative pollination behavior of Apis cerana F. and Apis mellifera L. on broccoli and their impact on seed production. Master Thesis Tribhuvan University. Institute of Agriculture and Animal Science, Rampur, Chitwan, Nepal.

[18] Emiru, B. 2002. Actual and Potential Contributions of Enclosure of Enhance Biodiversity in Dry Lands of Eastern Tigray with Particular Emphasis on Woody Plants, M.Sc. Thesis. ISSN 1402-201 X 2002; 70 (SIU), Sweden.

[19] Gallai, N., J. M. Salles, J. Settele, and B. E. Vaissiere. 2009. Economic valuation of the vulnerability of world agriculture confronted with pollinator decline. Ecol. Econ. 68: 810-821.

[20] Garratt, M.P.D., T. Breeze, N. Jenner, C. Polce, J.C. Biesmeijer and S.G. Potts. 2013. Avoiding a bad apple: insect pollination enhances fruit quality and economic value. Agric., Ecosys. Environ $84: 4-40$.

[21] Gulson, D., G.C. Lye, and B.Darvill. 2008. Decline and conservation of bumble bee, Ann.Rev.Entomol. 53:181-208

[22] Hogendoorn, K., F. Bartholomaeus and M.A. Keller. 2010. Chemical and sensory comparison of tomatoes pollinated by bees and by a pollination. J. Econ. Ent. 103:1286-1292.

[23] Isaacs, R. and A.K. Kirk. 2010. Pollination services provided to small and large highbush blueberry fields by wild and managed bees. J. of Appl. Eco. 47:841-849. 
[24] Jacobs, F., C. Simoens, D. Graaf and J. Deckers, 2006. Scope for non-wood forest products income generation from rehabilitation areas: focus on beekeeping. J. Drylands 1(2): 171-185.

[25] Mishra, R.C. 1997/1998. Perspectives in Indian apiculture. Agro. Botanica, HS Offset Printers, New Delhi, India.

[26] Neupane, K. R. 2001. Foraging preference of honeybee species to selected horticultural crops. M. Sc. Thesis submitted to IAAS, Rampur, Chitwan, Nepal.

[27] Partap, U. 1997. Bee flora of the Hindu-Kush-Himalayas: Inventory and management. ICIMOD, Kathmandu, Nepal.

[28] Partap, U. and T. Partap. 1997. Managed crop pollination. The missing dimension of mountain agriculture productivity. Discussion Paper No. MES 97/1. ICIMOD, Kathmandu, Nepal. pp 1-22.

[29] Partap, U. and T. Partap. 2002. Warning signal from the apple valley of the HKH: Productivity concerns and pollination problems. ICIMOD, Kathmandu, Nepal.

[30] Pokhrel, S. 2005. Behavior and Management of Domesticated and Wild honeybees (Apis spp.) in Chitwan, Nepal. Ph.D. dissertation. Tribhuvan University. 240 p.

[31] Pokhrel, S. 2006. Behavior and management of domesticated and wild honeybees (Apis spp.) in Chitwan, Nepal. Ph. D. dissertation, Department of Entomology, IAAS, Rampur, Chitwan, Nepal. 240

[32] Polumin, O. and A. Station. 1997. Flowers of Himalaya. Oxford University Press, ISBN 10: 0195641876

[33] Roldán, S. and S. Guerra. 2006. Quality fruit improvement in sweet pepper culture by bumble bee pollination. Scientia Horticulturae 110:160-166.

[34] Roselino, A. C., S.B. Santos, M. Hrncir and L.R. Bego. 2009. Differences between the quality of strawberries (Fragaria $\mathrm{x}$ ananassa) pollinated by the stingless bees Scaptotrigona aff. depilis and Nannotrigona testaceicornis. Genetics and Molecular Research 8:539-545.

[35] Shreshtha, K. 1998. Dictionary of Nepalese plant names. Mandala Book Point, Kathmandu, Nepal.

[36] Silveira F.A. (2004) Monitoring pollinating wildbees, In: Freitas B.M., Pereira J.O.P. (Eds.), Solitary bees: conservation, rearing and management for pollination, Imprensa Universitária UFC, Fortaleza.

[37] Thapa, R. B. 2006. Honeybees and other insect pollinators of cultivated crops: J. Inst. Agric. Anim. Sci. 27:1-23.

[38] Pokhrel, S, R., Thapa, F. P. Neupane, S. M. Shrestha. 2006. Absconding behavior and management of Apis cerana F. honeybee in Chitwan, Nepal. Journal of the Institute of Agriculture and Animal Science. 27: 77-86

[39] Thapa, R. B. 2002. Insect pollinators of some crop plants in Chitwan. NAHSON Bull.12/13:19-21.

[40] Winfree, R., I. Bartomeus and D. Cariveau. 2011. Native pollinators in anthropogenic habitats. Annual Review of Ecology, Evolution and Systematics 42:1-22.

[41] van-Engelsdorp, D., J.R.. Hayes., J., R.M. Underwood and J. Pettis. 2008. A Survey of Honey Bee Colony Losses in the U.S., Available: Spring 2008. PLoS ONE 3, e4071 in Jan12, 2015.

[42] UNEP. 1993. Global biodiversity. UNEP, Nerobi, Kenya.

[43] Williams, I.H. 1994. The dependence of crop production within the European Union on pollination by honey bees. Agric. Zool. Review. 6:229-257.

[44] Wilson, E. O. 1988. The current state of biodiversity. In : E. O Wilson and F. M. Peter (eds.) Biodiversity, National Academic Press, Washington DC, USA. 3-18 pp

*Corresponding author.

E-mail address: rijal_sp@yahoo.com 